\title{
Engineering a Robust Quantum Spin Hall State in Graphene via Adatom Deposition
}

\author{
Conan Weeks, ${ }^{1}$ Jun $\mathrm{Hu},{ }^{2}$ Jason Alicea, ${ }^{2}$ Marcel Franz, ${ }^{1}$ and Ruqian $\mathrm{Wu}^{2}$ \\ ${ }^{1}$ Department of Physics and Astronomy, University of British Columbia, Vancouver, British Columbia, V6T 1Z1 Canada \\ ${ }^{2}$ Department of Physics and Astronomy, University of California, Irvine, California 92697
}

(Received 17 May 2011; published 3 October 2011; corrected 30 March 2012)

\begin{abstract}
The 2007 discovery of quantized conductance in HgTe quantum wells delivered the field of topological insulators (TIs) its first experimental confirmation. While many three-dimensional TIs have since been identified, HgTe remains the only known two-dimensional system in this class. Difficulty fabricating HgTe quantum wells has, moreover, hampered their widespread use. With the goal of breaking this logjam, we provide a blueprint for stabilizing a robust TI state in a more readily available two-dimensional material—graphene. Using symmetry arguments, density functional theory, and tight-binding simulations, we predict that graphene endowed with certain heavy adatoms realizes a TI with substantial band gap. For indium and thallium, our most promising adatom candidates, a modest $6 \%$ coverage produces an estimated gap near $80 \mathrm{~K}$ and $240 \mathrm{~K}$, respectively, which should be detectable in transport or spectroscopic measurements. Engineering such a robust topological phase in graphene could pave the way for a new generation of devices for spintronics, ultra-low-dissipation electronics, and quantum information processing.
\end{abstract}

DOI: 10.1103/PhysRevX.1.021001 Subject Areas: Condensed Matter Physics, Graphene, Topological Insulators

\section{INTRODUCTION}

When certain physical properties of a system depend on global topology - and not on local details, such as disorder-then the system is said to realize a topological phase. By virtue of their universality, rooted in this independence of microscopic details, topological phases are both paradigmatically interesting and thought to possess seeds of important future technologies. Until rather recently, experimental studies of topological phases were confined to the quantum Hall effect, seen in high-quality twodimensional electron systems subjected to strong magnetic fields. The advent of topological insulators (TIs), a class of two- and three-dimensional nonmagnetic crystalline solids enjoying strong spin-orbit coupling [1-3], opened a fascinating new chapter in the field.

Two-dimensional TIs are commonly referred to as quantum spin-Hall (QSH) states [1-3] due to their similarity to integer quantum Hall liquids. A defining signature of a QSH state is the existence of gapless edge states which are protected from elastic backscattering and localization by time-reversal symmetry $(\mathcal{T})$. This feature, combined with the spin-filtered nature of the edge modes, renders such systems technologically very promising. Furthermore, when in contact with a superconductor, the edges are predicted to host Majorana fermions [4], which play an important role in topological quantum computation schemes [5]. The QSH state was first predicted to arise in graphene [6], although later it was realized that, due to

Published by the American Physical Society under the terms of the Creative Commons Attribution 3.0 License. Further distribution of this work must maintain attribution to the author(s) and the published article's title, journal citation, and DOI. carbon's small atomic number, spin-orbit coupling is too weak to produce an observable effect under realistic conditions. (First-principles calculations [7-11] on pristine graphene all predict sub-Kelvin gaps for this phase.) The QSH state was subsequently predicted in several other materials, including $\mathrm{HgTe}$ and InAs/GaSb quantum wells $[12,13]$ and bilayer bismuth [14]. While landmark experiments indeed observed this phase in HgTe [15], experimental activity on the QSH effect has remained limited by notorious practical difficulties associated with $\mathrm{HgTe}$ based structures.

Given the comparative ease with which graphene can be fabricated, it would be highly desirable to artificially enhance graphene's spin-orbit coupling strength to elevate the gap protecting the QSH state to observable levels. A practical means of doing so would pave the way to a new generation of QSH experiments accessible to a broad spectrum of researchers worldwide. Previous work has explored the possibility that strong interactions [16-18] or radiation [19] might drive graphene into a robust topological phase. Our goal in this manuscript is to explore a practical new route to this end. Specifically, we ask whether graphene can "inherit" strong spin-orbit coupling from a dilute concentration of heavy adatoms (whose innate spin-orbit coupling strength can be on the electron-volt scale) deposited randomly into the honeycomb lattice.

The basic principle underlying our proposal can be understood by considering processes in which an electron from graphene tunnels onto an adatom-whereupon it "feels" enormous spin-orbit coupling - and then returns to the graphene sheet. Such processes in effect locally enhance graphene's spin-orbit strength manyfold. A viable proposal must, however, address numerous competing effects. For instance, adatoms often form local magnetic 
moments [20] which potentially spoil the time-reversal symmetry protecting the QSH effect. Moreover, adatoms generically mediate both intrinsic and Rashba spin-orbit coupling. The latter is believed to be detrimental to the QSH phase [6], and previous work has indeed established that certain kinds of adatoms do generate substantial Rashba coupling in graphene that typically overwhelms the intrinsic contribution [21-23]. (As Ref. [23] showed, however, magnetic adatoms inducing strong Rashba coupling can induce an interesting "quantum anomalous Hall" state in graphene.) The adatoms may also favor competing, ordinary insulating states depending on their precise locations in the lattice. And finally, since spin-orbit coupling is generated nonuniformly in graphene, the stabilization of a QSH phase even in an otherwise ideal situation is unclear a priori.

After an extensive search using tight-binding and firstprinciples analyses, we have found that two elements, indium and thallium, are capable of stabilizing a robust QSH state in graphene. Neither element forms a magnetic moment, and although they do generate significant Rashba coupling, for symmetry reasons this remarkably does not suppress the QSH state. We find that gaps many orders of magnitude larger than that predicted in pure graphene can form even with coverages of only a few percent; for example, at $6 \%$ coverage, indium yields a gap on the order of $100 \mathrm{~K}$, while for thallium the gap approaches room temperature. These predictions revive graphene as a viable QSH candidate, and they can be verified by probing the gap and associated edge states using spectroscopic and conductance measurements.

\section{PHYSICS OF A SINGLE HEAVY ADATOM}

To set the stage, let us first briefly review the Kane-Mele model [6] describing pure, undoped graphene with spinorbit coupling. The Hamiltonian can be expressed as $H_{\mathrm{KM}}=H_{t}+H_{\mathrm{so}}$, where $H_{t}$ describes the usual nearestneighbor hopping and $H_{\text {so }}$ encodes intrinsic spin-orbit coupling. In terms of operators $c_{\mathbf{r} \alpha}^{\dagger}$ that add electrons with spin $\alpha$ to site $\mathbf{r}$ of the honeycomb lattice and Pauli matrices $s^{x, y, z}$ that act on the spin indices, $H_{t}$ and $H_{\text {so }}$ explicitly read

$$
\begin{gathered}
H_{t}=-t \sum_{\left\langle\mathbf{r r}^{\prime}\right\rangle}\left(c_{\mathbf{r}}^{\dagger} c_{\mathbf{r}^{\prime}}+\text { H.c. }\right), \\
H_{\text {so }}=\lambda_{\text {so }} \sum_{\left\langle\left\langle\mathbf{r} \mathbf{r}^{\prime}\right\rangle\right\rangle}\left(i \nu_{\mathrm{rr}^{\prime}} c_{\mathbf{r}}^{\dagger} s^{z} c_{\mathbf{r}^{\prime}}+\text { H.c. }\right) .
\end{gathered}
$$

Here and below, spin indices are implicitly summed whenever suppressed. In Eq. (2) the sum runs over secondnearest-neighbor lattice sites, and $\nu_{\mathbf{r r}^{\prime}}$ are signs that equal +1 , if an electron hops in the direction of the arrows in Fig. 1(c), and -1 otherwise. Thus $H_{\text {so }}$ describes "chiral" spin-dependent second-neighbor electron hopping. When $\lambda_{\mathrm{so}}=0$, the band structure exhibits the familiar gapless a)

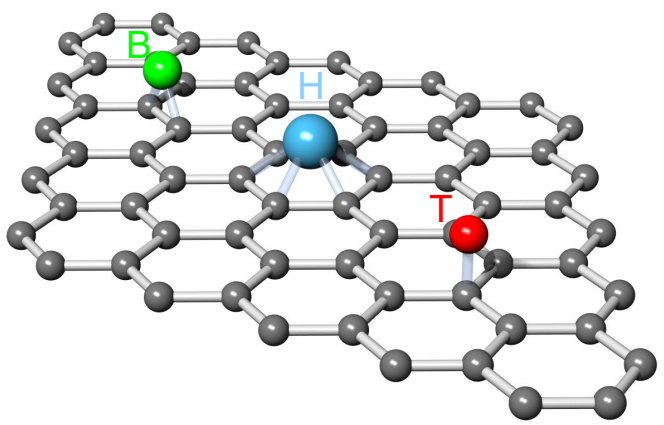

b)

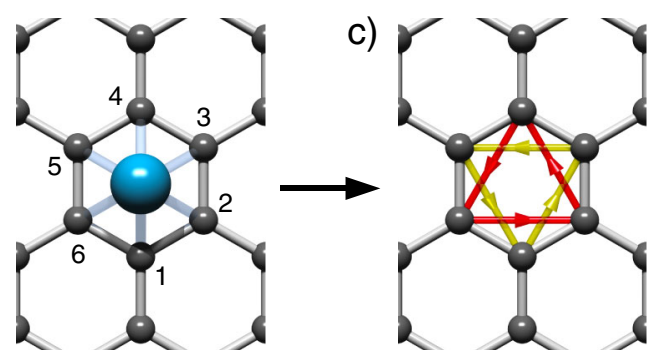

FIG. 1. Adatoms in graphene. (a) Depending on the element, adatoms favor either the high-symmetry "bridge" (B), "hollow" $(\mathrm{H})$, or "top" (T) position in the graphene sheet. (b) Detailed view of an H-position adatom, which is best suited for inducing the intrinsic spin-orbit coupling necessary for stabilizing the topological phase. The desired spin-orbit terms mediated by the adatom are illustrated in (c). Red and yellow bonds represent the induced second-neighbor imaginary hopping, whose sign is indicated by the arrows for spin-up electrons. For spin-down electrons, the arrows are reversed.

Dirac cones centered on momenta $\pm \mathbf{Q}$, resulting in semimetallic behavior. Turning on $\lambda_{\text {so }} \neq 0$ generates an energy gap [6] $\Delta=6 \sqrt{3}\left|\lambda_{\text {so }}\right|$ at the Dirac points, transforming the system into a (very fragile [7-11]) QSH insulator. Importantly, if mirror symmetry with respect to the graphene plane is broken, then Rashba coupling-which involves spin flips and thus breaks the $\mathrm{U}(1)$ spin symmetry enjoyed by $H$-will also be present [6]. Rashba coupling competes with the intrinsic spin-orbit term in pure graphene, and, beyond a critical value, it closes the gap and destroys the QSH state.

If heavy adatoms are to stabilize a more robust QSH phase in graphene, then, at a minimum, they should be nonmagnetic (to preserve $\mathcal{T}$ ) and modify the physics near the Dirac points primarily by inducing large intrinsic spinorbit coupling. The latter criterion leads us to focus on elements favoring the "hollow" $(\mathrm{H})$ position in the graphene sheet indicated in Fig. 1(a). Compared to the "bridge" (B) and "top" (T) positions, adatoms in the $\mathrm{H}$ position can most effectively mediate the spin-dependent second-neighbor hoppings present in the Kane-Mele model, while simultaneously avoiding larger competing effects such as local sublattice symmetry-breaking generated in the $\mathrm{T}$ case. 
Since H-position adatoms generically reside on one side of the graphene sheet, they will clearly mediate Rashba spin-orbit coupling as well, leading to a potentially delicate competition. If the adatoms' outer-shell electrons derive from $p$ orbitals, however, the induced intrinsic spin-orbit terms always dominate over the induced Rashba interactions. One can establish this key result by studying graphene with a single adatom of this type. To model this setup, we employ operators $d_{m \alpha}$ for the adatom states, with $m=0, \pm 1$ and $\alpha=\uparrow, \downarrow$ labeling the orbital and spin angular-momentum quantum numbers. The coupling of these orbitals to graphene is conveniently expressed in terms of the following operators:

$$
C_{m \alpha}=\frac{1}{\sqrt{6}} \sum_{j=1}^{6} e^{-i(\pi / 3) m(j-1)} c_{\mathbf{r}_{j} \alpha},
$$

where the sum runs over the six sites surrounding the adatom shown in Fig. 1(b). Guided by symmetry (see Appendix A), we consider the following minimal Hamiltonian for this single-adatom problem:

$$
\begin{gathered}
H=H_{g}+H_{a}+H_{c}, \\
H_{g}=H_{t}-\delta \mu \sum_{j=1}^{6} c_{\mathbf{r}_{j}}^{\dagger} c_{\mathbf{r}_{j}}, \\
H_{a}=\sum_{m=0, \pm 1} \epsilon_{|m|} d_{m}^{\dagger} d_{m}+\Lambda_{\mathrm{so}}\left(d_{1}^{\dagger} s^{z} d_{1}-d_{-1}^{\dagger} s^{z} d_{-1}\right) \\
+\sqrt{2} \Lambda_{\mathrm{so}}^{\prime}\left(d_{0}^{\dagger} s^{-} d_{-1}+d_{0}^{\dagger} s^{+} d_{1}+\text { H.c. }\right), \\
H_{c}=-\sum_{m=0, \pm 1}\left(t_{|m|} C_{m}^{\dagger} d_{m}+\text { H.c. }\right),
\end{gathered}
$$

with $s^{ \pm}=\left(s^{x} \pm i s^{y}\right) / 2$. Here $H_{g}$ represents the nearestneighbor hopping model of graphene supplemented by a chemical potential $\delta \mu$ for the six sites surrounding the adatom. Physically, a nonzero $\delta \mu$ leads to an excess electron density at those sites, screening any net charge from the adatom. Crystal-field effects and spin-orbit coupling split the adatom $p$ orbitals through the $\epsilon_{|m|}$ and $\Lambda_{\text {so }}$, $\Lambda_{\text {so }}^{\prime}$ terms in $H_{a}$. Finally, $H_{c}$ allows electrons to tunnel between the adatom and its neighboring carbon sites. All couplings in $H$ are real except $t_{1}$, which is pure imaginary. Note also that we have ignored the exceedingly weak spin-orbit terms that couple the electrons in graphene directly, as well as Coulomb interactions for the adatom. Exclusion of these latter terms is justified for the nonmagnetic adatoms that we will consider below.

Recalling that intrinsic spin-orbit coupling in graphene conserves the $S^{z}$ spin component while Rashba coupling does not, one can readily understand how the adatom mediates both kinds of interactions by viewing the tunnelings in $H_{c}$ perturbatively. For example, when an electron hops onto the $m=0$ orbital via $t_{0}$, flips its spin through the
$\Lambda_{\text {so }}^{\prime}$ coupling, and then hops back to the honeycomb lattice via $t_{1}$, spin-orbit coupling of the Rashba type is locally generated in graphene. In sharp contrast to the situation for the Kane-Mele model, however, Rashba terms mediated in this fashion are irrelevant for the low-energy physics. This crucial feature can be understood by Fourier transforming $C_{0 \alpha}$; remarkably, the components with Dirac-point momenta $\pm \mathbf{Q}$ vanish identically. More physically, electrons near the Dirac points interfere destructively when hopping onto the $m=0$ adatom orbital. (See Appendixes A and D for complementary perspectives.) We stress that this argument holds only for $p$ orbitals. If the relevant adatom orbitals carry higher angular momentum, spin-flip processes which do affect the Dirac points will generically appear. The induced Rashba terms may still be subdominant, although whether this is the case depends on details of the Hamiltonian, unlike the situation for $p$ orbitals.

In contrast, $S^{z}$-conserving events whereby an electron hops both onto and then off an $m= \pm 1$ orbital via $t_{1}$ locally mediate the desired intrinsic spin-orbit interactions. No obstruction exists for tunneling onto the $m= \pm 1$ orbitals via $t_{1}$, so these processes can effectively modify the physics near the Dirac points. It is important to note, however, that $t_{1}$ hopping mediates additional couplings as well, so the dominance of these spin-orbit terms remains unclear. Nevertheless, given the high symmetry preserved by the $\mathrm{H}$-site adatom, none of the conventional brokensymmetry gapped phases of graphene-such as chargedensity wave or "Kekule" orders-is obviously favored here, so it is reasonable to expect these additional terms to play a relatively minor role. We explicitly confirm this intuition in the multiadatom situation, to which we now turn.

\section{PERIODIC ADATOM CONFIGURATIONS}

As a first step in understanding the multiadatom case, we examine a periodic system with one adatom residing in a large $N \times N$ supercell. This situation allows us to utilize density functional theory (DFT) to ascertain suitable heavy elements and obtain a quantitative understanding of their effects on graphene. (See Appendix B for computational details.) To ensure large spin-orbit coupling, we focused on elements in rows five and six of the periodic table, including In, Sn, Sb, Te, I, La, Hf, Pt, $\mathrm{Au}, \mathrm{Hg}, \mathrm{Tl}, \mathrm{Pb}$ and $\mathrm{Bi}$. For each element, we calculated the total energy in the three adsorption geometries shown in Fig. 1(a) along with the adatom's spin moment. Our calculations reveal that two elements-indium (atomic number $Z=49$ ) and thallium $(Z=81)$ - satisfy our criteria of both favoring the high-symmetry $\mathrm{H}$ position and being nonmagnetic. Furthermore, both elements exhibit partially filled $p$ shells, ensuring that the Rashba coupling they mediate in graphene is benign at the Dirac points.

It is instructive to first examine the electronic properties of indium on graphene in a $4 \times 4$ supercell, without 
(a) DFT, no SOC (In)

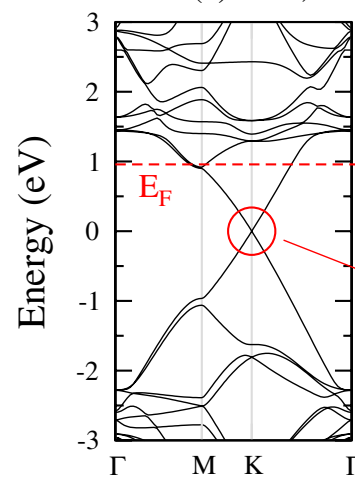

(d) DFT, no SOC (Tl)

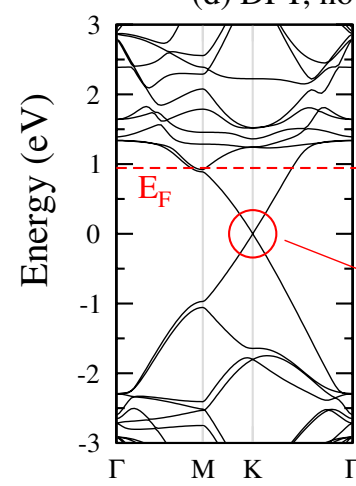

(b) DFT, with SOC (In)

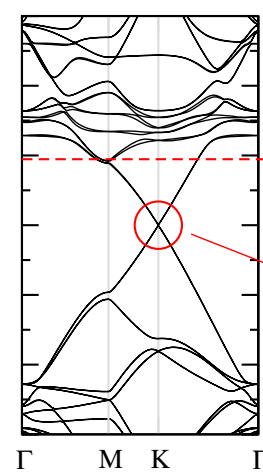

(e) DFT, with SOC (Tl)

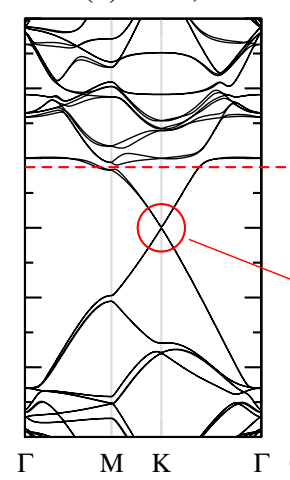

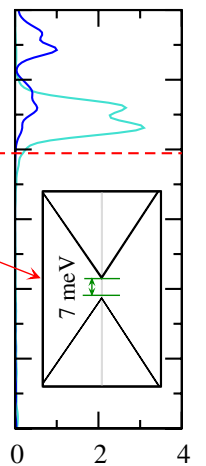

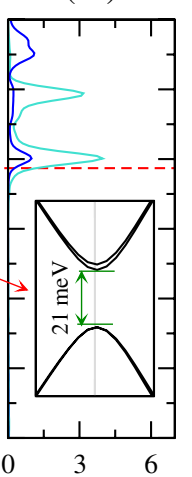

(c) tight-binding, with SOC (In)

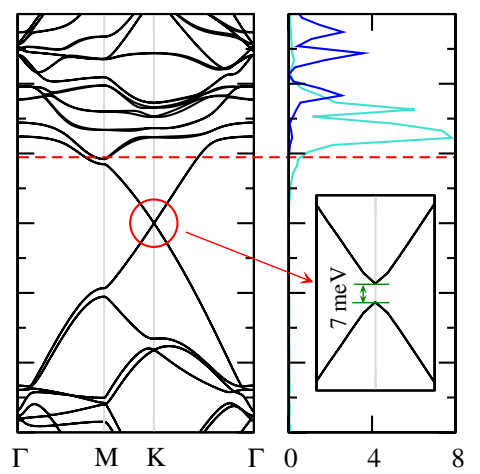

(f) tight-binding, with SOC (Tl)

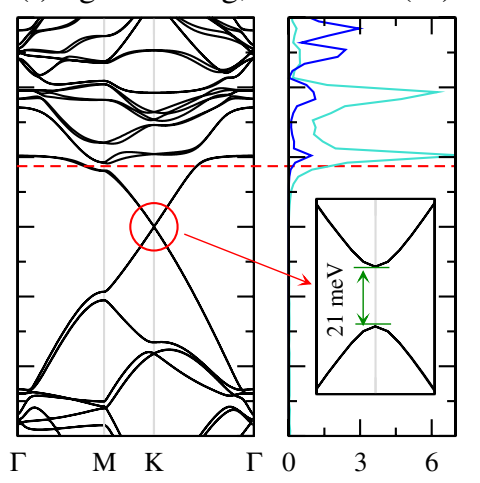

FIG. 2. Band structure and the adatom local density of states (LDOS). All data correspond to one adatom in a $4 \times 4$ supercell, with the upper row corresponding to indium and the lower row corresponding to thallium. The left panels in (a) and (d) correspond to the band structure and LDOS computed using DFT without spin-orbit coupling. The horizontal dashed red lines indicate the Fermi level $\left(E_{\mathrm{F}}\right)$, which shifts due to electron-doping from the adatoms. Insets zoom in on the band structure near the K point within an energy range -35 to $35 \mathrm{meV}$, showing that, without spin-orbit interactions, neither indium nor thallium opens a gap at the Dirac points. The central panels in (b) and (e) are the corresponding DFT results including spin-orbit coupling. Remarkably, in the indium case, a gap of $7 \mathrm{meV}$ opens at the Dirac points, while, with thallium, the gap is larger still at $21 \mathrm{meV}$. Finally, the right panels in (c) and (f) were obtained using the tight-binding model described in Sec. III.

spin-orbit coupling. As Fig. 2(a) illustrates, the Dirac cones characteristic of pure graphene indeed remain masslessdespite the reduced translation symmetry, conventional gapped phases are not stabilized here, consistent with the intuition developed in the single-adatom case above. Indium does, however, electron-dope graphene and shifts the Fermi level $E_{\mathrm{F}}$ to $0.95 \mathrm{eV}$ above the Dirac points. From the adatom's local density of states (LDOS) displayed in Fig. 2(a), one can see that indium's $5 p$ orbitals lie almost entirely above $E_{\mathrm{F}}$, implying that the $5 p$ electron in neutral indium nearly completely transfers to graphene. (The charge of an indium adatom is $+0.8 e$ from the Bader charge division scheme.) Note that the relatively diffuse $p_{z}$ LDOS indicates that this orbital hybridizes more strongly with graphene compared to the $p_{x, y}$ orbitals. Replacing indium with thallium, again without spin-orbit coupling, leads to the band structure and LDOS shown in Fig. 2(d). Clearly the electronic structure is modified very little by this substitution; importantly, the Dirac cones remain massless with thallium as well.
Thus any gap opening at the Dirac points must originate from spin-orbit coupling. Figure 2(b) displays the band structure and LDOS for spin-orbit-coupled indium on graphene. Note the sizable spin-orbit splitting in the LDOS for the $p_{x, y}$ orbitals. More remarkably, a gap $\Delta_{\text {so }} \approx 7 \mathrm{meV}$ now appears at the Dirac points, which already exceeds the spin-orbit-induced gap in pure graphene [7-11] by several orders of magnitude. The analogous results for thalliumwhose atomic mass is nearly twice that of indium - are still more striking. As Fig. 2(e) illustrates, $p$-orbital splittings of order $1 \mathrm{eV}$ are now evident in the LDOS, and a gap $\Delta_{\text {so }} \approx 21 \mathrm{meV}$ opens at the Dirac points. We emphasize that these results apply for adatom coverages of only $6.25 \%$. To explore the dependence of $\Delta_{\text {so }}$ on the adatom coverage, we additionally investigated systems with one adatom in $5 \times 5,7 \times 7$, and $10 \times 10$ supercells. (For $N \times N$ supercells with $N$ a multiple of 3 , the Dirac points reside at zero momentum and can thus hybridize and gap out even without spin-orbit coupling. We therefore ignore such geometries.) The values of $\Delta_{\text {so }}$ along with the Fermi level $E_{\mathrm{F}}$ computed for the coverages we studied appear 
in Fig. 3. Quite naturally, the gap decreases as one reduces the coverage, as does the Fermi level since fewer electrons are donated to graphene at lower adatom concentrations. It is worth highlighting that the gap decreases sublinearly and that a sizable $\Delta_{\text {so }} \approx 10 \mathrm{meV}$ remains even with a mere $2.04 \%$ thallium concentration.

Since spin-orbit coupling clearly underlies the gap onset, it is tempting to associate the insulating regime opened by the adatoms with a QSH state. To verify this expectation, we appeal to tight-binding simulations of the $4 \times 4$ supercell geometry. We model the system by a Hamiltonian $H_{4 \times 4}$ consisting of uniform hopping for graphene (including weak second- and third-neighbor tunneling) supplemented by the additional local terms in Eq. (4) at each adatom. Figure 2(c) displays the tight-binding band structure and adatom LDOS for indium with the following parameters: first, second, and third-neighbor graphene hoppings $t=2.82 \mathrm{eV}, t^{\prime}=$ $0.22 \mathrm{eV}$, and $t^{\prime \prime}=0.2 \mathrm{eV} ; \delta \mu=0.5 \mathrm{eV} ; \epsilon_{0}=2.5 \mathrm{eV}$, $\epsilon_{1}=1.8 \mathrm{eV} ; \quad t_{0}=2 \mathrm{eV}, \quad i t_{1}=0.95 \mathrm{eV} ; \quad$ and $\Lambda_{\mathrm{so}}=$ $\Lambda_{\mathrm{so}}^{\prime}=0.1 \mathrm{eV}$. The corresponding data for thallium appear in Fig. 2(f); the parameters are the same as for indium, except for $\epsilon_{1}=1.9 \mathrm{eV}$ and $\Lambda_{\mathrm{so}}=\Lambda_{\mathrm{so}}^{\prime}=$ $0.31 \mathrm{eV}$. The good agreement with DFT provides strong evidence that the underlying physics of the spin-orbitcoupled system is well-captured by our tight-binding model. With this result in hand, one can then demonstrate the topological nature of the insulating regime by showing that the Dirac-point gap remains nonzero upon adiabatically deforming the Hamiltonian to the Kane-Mele model. This can be achieved by defining a new Hamiltonian $H(\lambda)=(1-\lambda) H_{4 \times 4}+\lambda H_{\mathrm{KM}}$ that interpolates between our adatom model at $\lambda=0$ and the KaneMele model at $\lambda=1$. For either indium or thallium parameters, the Dirac-point gap never closes as $\lambda$ varies from 0 to 1 (see Appendix $\mathrm{C}$ for details). Thus either element can indeed stabilize a robust QSH state in graphene, at least when periodically arranged.

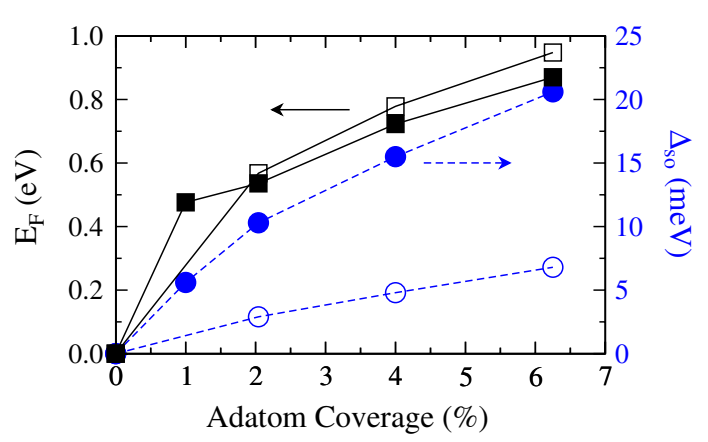

FIG. 3. Coverage effects. Spin-orbit-induced gap $\Delta_{\text {so }}$ (circles) opened at the Dirac points and Fermi level $E_{\mathrm{F}}$ (squares) measured relative to the center of the gap for different indium and thallium adatom coverages. The open and filled symbols represent data for indium and thallium, respectively.

\section{RANDOMLY DISTRIBUTED ADATOMS}

In an experiment, adatoms will likely occupy random $\mathrm{H}$-site locations in a graphene sheet that is of course not pristine. It is therefore important to understand the impact of such randomness and disorder on the stability of the topological phase that was found above in pure graphene with periodic adatoms. To address general features of the problem in a way that reduces its computational complexity, we will describe the disordered, random-adatom system using the following graphene-only Hamiltonian,

$$
H=H_{t}+\sum_{I} \delta H_{I}-\sum_{\mathbf{r}} \delta \mu_{\mathbf{r}} c_{\mathbf{r}}^{\dagger} c_{\mathbf{r}}
$$

Here $\delta \mu_{\mathrm{r}}$ represents a random on-site potential arising, e.g., from the substrate (and not from the adatoms). In the second term, $I$ labels the random plaquettes occupied by adatoms and

$$
\begin{aligned}
\delta H_{I}= & -\delta \mu \sum_{\mathbf{r} \in I} c_{\mathbf{r}}^{\dagger} c_{\mathbf{r}}+\lambda_{\text {so }} \sum_{\left\langle\left\langle\mathbf{r r}^{\prime}\right\rangle\right\rangle \in I}\left(i \nu_{\mathbf{r r}^{\prime}} c_{\mathbf{r}^{\prime}}^{\dagger} s^{z} c_{\mathbf{r}^{\prime}}+\text { H.c. }\right) \\
& +i \lambda_{R} \sum_{\mathbf{r}, \mathbf{r}^{\prime} \in I} c_{\mathbf{r}}^{\dagger}\left(\mathbf{s} \times \mathbf{d}_{\mathbf{r r}^{\prime}}\right)_{z} c_{\mathbf{r}^{\prime}},
\end{aligned}
$$

with $\mathbf{d}_{\mathbf{r r}^{\prime}}=\left(\mathbf{r}-\mathbf{r}^{\prime}\right) /\left|\mathbf{r}-\mathbf{r}^{\prime}\right|$. The first term in $\delta H_{I}$ describes the chemical potential that screens charge from the adatoms, while the last two terms capture the local intrinsic $\left(\lambda_{s o}\right)$ and Rashba $\left(\lambda_{R}\right)$ spin-orbit couplings induced by electrons hopping from graphene to an adatom and back, as discussed in Sec. II. Formally, these spin-orbit terms can be derived by integrating out perturbatively the adatom degrees of freedom in the Hamiltonian (5)-(7); see Appendix D. It is important to notice that, unlike the conventional nearest-neighbor Rashba term considered in Ref. [6] for pristine graphene, the adatom-generated Rashba term connects all sites in the hexagon. Such a "hexagon Rashba term" has the property of vanishing at the Dirac points, as already argued in Sec. II. The adatom also induces other symmetry-allowed terms, such as further-neighbor spin-independent hoppings, which we disregard because they either are weak or do not lead to qualitative changes in the results reported below.

To study the robustness of the QSH phase in the presence of random adatoms and disorder, we probe for the signature gapless edge states by simulating the classic twoterminal conductance measurement in the geometry of Fig. 4(a). Since "zig-zag" edges are known to support gapless edge states (of a qualitatively different kind) even in pristine graphene, to avoid complications we focus here on graphene strips with "armchair" edges. We emphasize, however, that the results reported below remain valid for arbitrary edge configurations, as long as the bulk remains in the topological phase. Within the Landauer-Büttiker formalism [24,25], we employ the recursive Green's function method [26] to evaluate the conductance $G$ of a length $L$, width $W$ graphene strip as we vary the Fermi energy. Consider first the simplest random-adatom setup with 
(a)

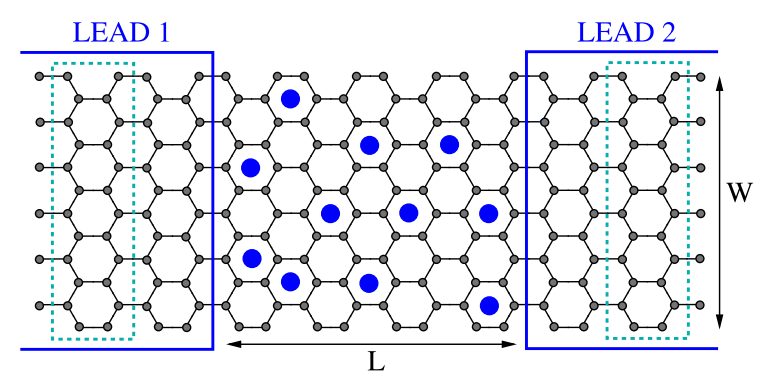

(c)

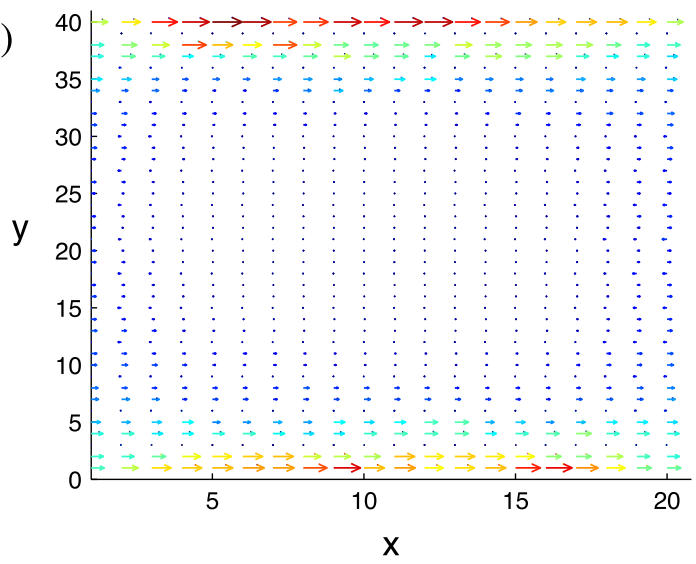

(b)

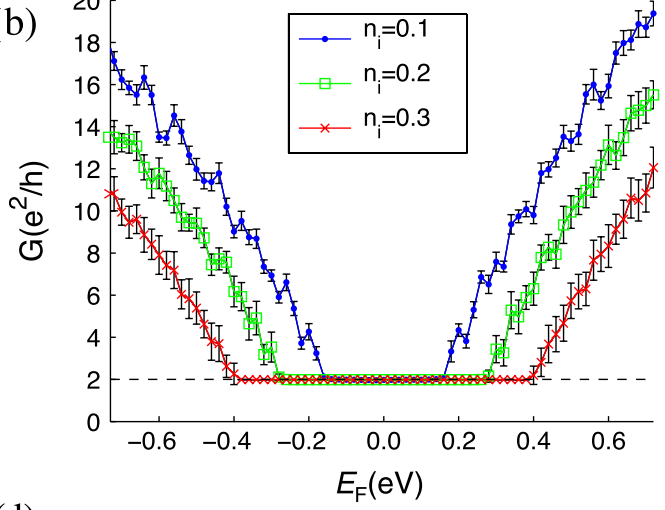

(d)

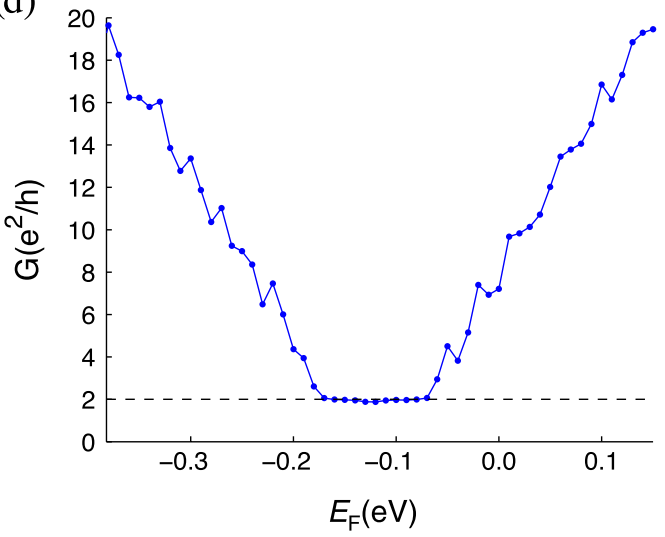

FIG. 4. Transport. (a) Configuration used to determine the two-terminal conductance. Semi-infinite clean graphene leads are connected to the sample, which has adatoms distributed randomly across it. The length $L$ of the system is set by the number of columns (indicated by dotted lines). The width $W$ refers to the number of sites along either edge of the figure, corresponding to the number of unit cells of an armchair nanoribbon required to create an individual column. (b) Conductance $G$ as a function of the Fermi energy $E_{\mathrm{F}}$, averaged over 40 independent random-adatom realizations at different concentrations $n_{i}=0.1,0.2,0.3$ for a system of size $W=80$ and $L=40$ with $\lambda_{\mathrm{so}}=0.1 t$. (c) Current distribution across a sample of size $W=40$ and $L=20$ at $n_{i}=0.2, \lambda_{\mathrm{so}}=0.1 t$ and $E_{\mathrm{F}}=0.15 \mathrm{eV}$. The magnitude of the current is represented by both the arrow size and color. (d) Conductance for the largest simulated system size using realistic parameters for thallium adatoms $\left(\lambda_{\mathrm{so}}=0.02 t\right.$ and $\left.\delta \mu=0.1 t\right)$ estimated from DFT data. Here $W=200$, $L=100$, and the coverage is $n_{i}=0.15$. In all panels we take $t=2.7 \mathrm{eV}$.

$\delta \mu_{\mathbf{r}}=0$ (corresponding to an otherwise clean graphene sheet), $\delta \mu=0$, and $\lambda_{R}=0$. Figure 4(b) illustrates the conductance for this case at several adatom concentrations $n_{i}$ and model parameters indicated in the caption. A $2 e^{2} / h$ plateau with width proportional to $n_{i}$ clearly emerges, strongly suggesting the onset of a bulk mobility gap and quantized ballistic conduction by edge states. This picture is corroborated by Fig. 4(c), which displays the current distribution for $E_{\mathrm{F}}=0.15 \mathrm{eV}$ across a smaller system size (chosen for clarity).

These results establish that, in principle, adatoms need not be periodic to stabilize a QSH phase, although the parameters employed were unrealistic. To make contact with our DFT results, we also considered a system with $\lambda_{\text {so }}=0.02 t$ and $\delta \mu=0.1 t$, which would yield a similarbulk mobility gap to that seen in our thallium simulations if the adatom coverages were the same. While finite-size effects prevent us from studying the low-adatom coverages considered in Sec. III, Fig. 4(d) shows that, for $n_{i}=0.15$, a robust conductance plateau indeed persists for these more realistic parameter values. (At much lower coverages, the width of the edge states approaches the system sizes we are able to simulate.) We have also studied the effects of the hexagon Rashba term and residual on-site potential disorder on the stability of the QSH phase (see Appendix E for details). Even a relatively large $\lambda_{R} \sim 2 \lambda_{\text {so }}$ has a weak effect on the width of the conductance plateau, as expected given our analysis in Sec. II. With uncorrelated on-site disorder $\delta \mu_{\mathbf{r}}$, the topological phase is also remarkably robust $-\delta \mu_{\mathrm{r}}$ can fluctuate on the scale of $t$ while degrading the plateau only marginally. The topological phase is more sensitive, however, to correlated disorder which is likely more relevant experimentally. In this case, the conductance plateau survives only when $\delta \mu_{\mathbf{r}}$ varies on a scale smaller roughly than the mobility gap for the clean case. For one to observe the QSH phase experimentally, the chemical potential should therefore fluctuate on scales smaller than the $\sim 10 \mathrm{meV}$ gaps we predicted here. While this might be difficult to achieve with graphene on a standard $\mathrm{SiO}_{2}$ substrate, recent experiments using 
hexagonal boron nitride substrates [27] show disorder energy scales as low as $15 \mathrm{~K}$, which should be sufficient to observe the QSH gap.

\section{RELATION TO PREVIOUS WORK}

Since numerous recent studies have explored the possibility of employing adatoms to control graphene's electronic properties, here we briefly comment on the distinctions between our proposal and related earlier works. Castro Neto and Guinea [21] previously argued that graphene's spin-orbit strength can be greatly enhanced by adatom deposition, although the physical mechanism and nature of induced spin-orbit coupling is quite different from what we considered here. The enhancement discussed in Ref. [21] stemmed from structural changes in the honeycomb lattice induced by a T-position adatom (such as hydrogen whose innate spin-orbit coupling is weak). We explicitly neglected such contributions, as our DFT simulations demonstrated that the adatoms we considered only weakly affect the carbon-carbon bonds. Perhaps even more important, the effect was discussed solely in the context of enhanced Rashba spin-orbit interactions, which do not stabilize a QSH state in graphene.

More recently, Abdelouahed et al. [22] examined the influence of heavy Au adatoms on graphene's electronic structure using first-principles calculations. These authors considered dense coverages, with 1 T-position adatom residing above each carbon site on graphene's A sublattice, and found substantial enhancement of graphene's Rashba coupling to values of order $10 \mathrm{meV}$. Again, this type of induced-spin-orbit coupling is not suitable for driving a QSH state, and, in any case, this geometry is very likely to produce only a topologically trivial band insulator due to explicit breaking of sublattice symmetry by the adatoms. (Lighter Ni adatoms were also studied but were found to modify graphene's spin-orbit coupling only very weakly.)

Closest in spirit to our study is the very interesting work of Qiao et al. [23], who considered the possibility that adatoms can stabilize another topological phase in graphene-a quantum anomalous Hall state. Unlike the QSH phase, this state breaks time-reversal symmetry and exhibits chiral edge modes leading to a nontrivial Hall conductivity as in the integer quantum Hall effect. Thus the requirements for stabilizing a quantum anomalous Hall phase in graphene are quite different. Qiao et al. showed that this can be accomplished by a combination of strong Rashba spin-orbit coupling and an exchange-induced Zeeman field. They further demonstrated via firstprinciples calculations that $6.25 \%$ coverage of periodically arranged iron adatoms (which form magnetic moments) provide both ingredients and generate a quantum anomalous Hall phase protected by a $5.5 \mathrm{meV}$ gap. Note that, while iron favors the $\mathrm{H}$ site in the same way that indium and thallium do, its outer-shell electrons derive from $d$ rather than $p$ orbitals. Thus our argument for the irrelevance of Rashba spin-orbit coupling at the Dirac points does not apply to iron adatoms, consistent with the numerical findings of Ref. [23]. Although the gap produced by iron is smaller than that produced by indium and especially thallium at the same coverage, a promising feature of their proposal is that iron adatoms do not alter the Fermi level.

We also wish to point out a connection of our work to "topological Anderson insulators"- - systems that are nontopological in the clean limit but acquire nontrivial topology when disordered. Such states were first predicted in Ref. [28] and explored further in several subsequent studies [29-32]. The robust QSH state that can emerge upon randomly depositing dilute adatoms onto pure graphene (which by itself is a nontopological semimetal) can be viewed as an example of a topological Anderson insulator. Note, however, that this categorization is somewhat loose, as, in fact, this phase smoothly connects to the topological phase of the pure Kane-Mele model [6], which has no disorder (see also Ref. [32]). Nevertheless, this viewpoint is useful in that it highlights the remarkable fact that disorder, with suitable properties, can provide a practical avenue to generating topological phases.

\section{DISCUSSION}

In this paper we have demonstrated that dilute heavy adatoms - indium and thallium, in particular-are capable of stabilizing a robust QSH state in graphene, with a band gap exceeding that of pure graphene by many orders of magnitude. Aside from fabrication ease, another virtue graphene possesses as a potential QSH system is the large number of experimental probes available in this material. Angle-resolved photoemission spectroscopy, for instance, actually benefits from electron doping by the adatoms and thus could be employed to detect the onset of the Dirac-point gap at arbitrary coverages. Scanning tunneling microscopy would be similarly well-suited for probing the bulk gap, the spatial structure of the LDOS around an adatom, and even the topologically protected gapless edge modes.

Detecting the edge states via transport will be more challenging, since this would require repositioning the Fermi level inside of the bulk gap. Nevertheless, the technology for doping graphene by the requisite amount does exist (see, e.g., Refs. [33-37]). Although the most commonly used ion-liquid techniques [33-35] work best near room temperature, recently developed approaches employing ferroelectric substrates [36] and solid polymer electrolytes [37] show comparable results and remain applicable down to cryogenic temperatures. The requisite doping may also be achievable by introducing additional highelectronegativity adatoms that absorb electrons. A quantum spin-Hall phase in an appropriately doped graphene sample can be detected through the classic two-terminal conductance measurement akin to the historic observation 
performed in $\mathrm{HgTe}$ quantum wells [15]. A more complete characterization of the topological phase can be obtained through nonlocal edge state [38] and magnetotransport measurements which provide unambiguous evidence for topologically protected helical edge states $[39,40]$.

Fulfilling Kane and Mele's original vision by artificially turning graphene into a strong spin-orbit system could have remarkable technological implications. Among these, the possibility of employing the protected edge states for realizing topological superconductivity, Majorana fermions, and related phenomena such as non-Abelian statistics and "fractional Josephson effects" are particularly tantalizing [4]. Interestingly, one of the primary requirements for observing these phenomena-generating a superconducting proximity effect in graphene-has already been achieved by numerous groups [41-44]. These exciting possibilities, along with potential spintronics and lowdissipation devices, provide strong motivation for pursuing the line of attack we have introduced for stabilizing a robust QSH state in graphene.

\section{ACKNOWLEDGMENTS}

It is a pleasure to acknowledge helpful discussions with Jim Eisenstein, Ilya Elfimov, Josh Folk, Erik Henriksen, Roland Kawakami, Shu-Ping Lee, Gil Refael, Shan-Wen Tsai, and Amir Yacoby. J. A. gratefully acknowledges support from the National Science Foundation through Grant No. DMR-1055522. Work at UBC was supported by NSERC and CIfAR. J. H. and R. W. acknowledge support from the DOE-BES (Grant No. DE-FG0205ER46237) and computing time on NERSC supercomputers.

\section{APPENDIX A: DISCUSSION OF THE EFFECTIVE HAMILTONIAN FOR GRAPHENE WITH A SINGLE ADATOM}

In this section, we will consider a sheet of graphene located in the $(x, y)$ plane, with a single adatom sitting at the $\mathrm{H}$ position (recall Fig. 1). An important feature of such an adatom is the high symmetry that it preserves compared to the cases where the adatom resides at the $\mathrm{B}$ or $\mathrm{T}$ positions. In particular, the following symmetries remain here: $\pi / 3$ rotation $\left(R_{\pi / 3}\right)$ and $x$ reflection $\left(R_{x}\right)$ about the adatom site, as well as time reversal $(\mathcal{T})$. Note that, since the adatom is displaced vertically out of the $(x, y)$ plane, mirror symmetry with respect to the graphene plane $\left(R_{z}\right)$ is broken. It will nevertheless be useful below to understand how operators transform under $R_{z}$, since this will give us insight into which couplings mediate intrinsic versus Rashba spin-orbit coupling.

We will continue to assume that $p$ orbitals form the active states for the adatom. Also as in the main text, we employ operators $d_{m \alpha}^{\dagger}$, which add electrons with spin $\alpha$, and orbital angular momentum quantum number $m=0$, \pm 1 to the adatom, and operators $c_{\mathbf{r} \alpha}^{\dagger}$, which add electrons with spin $\alpha$ to site $\mathbf{r}$ of graphene's honeycomb lattice. These operators transform under $R_{\pi / 3}$ according to

$$
\begin{gathered}
R_{\pi / 3}: c_{\mathbf{r}} \rightarrow e^{i(\pi / 6) s^{z}} c_{\mathbf{r}^{\prime}}, \\
R_{\pi / 3}: d_{m} \rightarrow e^{i(\pi / 6) s^{z}} e^{i(m \pi / 3)} d_{m},
\end{gathered}
$$

where $\mathbf{r}^{\prime}$ corresponds to $\mathbf{r}$ rotated by $\pi / 3$. Note that, here and below, both $c_{\mathbf{r}}$ and $d_{m}$ are considered to be twocomponent objects, with the upper and lower components corresponding to spin up and down, respectively. Similarly, under $R_{x}$, we have

$$
\begin{gathered}
R_{x}: c_{(x, y)} \rightarrow e^{i(\pi / 2) s^{x}} c_{(-x, y)}, \\
R_{x}: d_{m} \rightarrow e^{i(\pi / 2) s^{x}} d_{-m} .
\end{gathered}
$$

Time reversal, which is antiunitary, sends

$$
\begin{gathered}
\mathcal{T}: c_{\mathbf{r}} \rightarrow e^{i(\pi / 2) s^{y}} c_{\mathbf{r}}, \\
\mathcal{T}: d_{m} \rightarrow(-1)^{m} e^{i(\pi / 2) s^{y}} d_{-m} .
\end{gathered}
$$

Note that the $(-1)^{m}$ factor above arises because one conventionally defines spherical harmonics such that $\left[Y_{\ell}^{m}\right]^{*}=$ $(-1)^{m} Y_{\ell}^{-m}$. Finally, the operators transform under $R_{z}$ as

$$
\begin{gathered}
R_{z}: c_{\mathbf{r}} \rightarrow-e^{i(\pi / 2) s^{z}} c_{\mathbf{r}}, \\
R_{z}: d_{m} \rightarrow(-1)^{1-m} e^{i(\pi / 2) s^{z}} d_{m} .
\end{gathered}
$$

In the last equation, the factor of $(-1)^{1-m}$ reflects properties of spherical harmonics under $z \rightarrow-z$.

Our aim now is to construct a minimal tight-binding model for graphene with a single $\mathrm{H}$-position adatom. As in Sec. II, we write the Hamiltonian as $H=H_{g}+H_{a}+H_{c}$, where $H_{g}$ involves only the carbon degrees of freedom, $H_{a}$ involves only the adatom degrees of freedom, and $H_{c}$ couples the two. Consider first $H_{a}$. The most general quadratic form for the adatom Hamiltonian can be written

$$
H_{a}=\sum_{\alpha=0, x, y, z} \sum_{m, m^{\prime}=-1}^{1} u_{m, m^{\prime}}^{\alpha} d_{m}^{\dagger} s^{\alpha} d_{m^{\prime}},
$$

where $s^{0}$ is the identity, $s^{x, y, z}$ are Pauli matrices, and $u_{m, m^{\prime}}^{\alpha}=\left[u_{m^{\prime}, m}^{\alpha}\right]^{*}$ due to Hermicity. Using the transformation rules obtained above, one can show that symmetry strongly constrains the allowed $u_{m, m^{\prime}}^{\alpha}$ terms. For example, in the $\alpha=0$ sector, $R_{\pi / 3}$ implies that $u_{m, m^{\prime}}^{0}$ must vanish except when $m=m^{\prime}$, while $R_{x}$ further requires that $u_{m, m}^{0}=u_{-m,-m}^{0}$. (Time reversal constrains these couplings no further.) The $\alpha=x, y, z$ terms can be similarly constrained, leading to the expression

$$
\begin{aligned}
H_{a}= & \sum_{m=0, \pm 1} \epsilon_{|m|} d_{m}^{\dagger} d_{m}+\Lambda_{\mathrm{so}}\left(d_{1}^{\dagger} s^{z} d_{1}-d_{-1}^{\dagger} s^{z} d_{-1}\right) \\
& +\sqrt{2} \Lambda_{\mathrm{so}}^{\prime}\left(d_{0}^{\dagger} s^{-} d_{-1}+d_{0}^{\dagger} s^{+} d_{1}+\text { H.c. }\right)
\end{aligned}
$$

of Eq. (6). 
Let us elaborate on the physics here in greater detail. If the adatom were in isolation, continuous rotation symmetry would further restrict $\epsilon_{|m|}$ to be independent of $m$ and require that $\Lambda_{\mathrm{so}}^{\prime}=\Lambda_{\mathrm{so}}$. In this case, the $\Lambda_{\mathrm{so}}, \Lambda_{\mathrm{so}}^{\prime}$ terms above simply encode an isotropic $\mathbf{L} \cdot \mathbf{S}$ spin-orbit interaction that split the orbitals into a lower $j=1 / 2$ doublet and a $j=3 / 2$ quadruplet that is higher in energy by $3 \Lambda_{\mathrm{so}}$. Crystal-field effects arising from the graphene environment lead to $m$-dependence of $\epsilon_{|m|}$ and allow $\Lambda_{\text {so }}^{\prime}$ to differ from $\Lambda_{\mathrm{so}}$. Note also that we have assumed that the adatom's $s$ orbitals (and all other inner levels) are far in energy from the Dirac points and can be safely neglected. This is indeed justified by our supercell DFT calculations for both indium and thallium, where the $5 s$ and $6 s$ electrons contribute significantly to the density of states only at several $\mathrm{eV}$ below the Dirac points. We have further ignored fourfermion interaction terms in $H_{a}$. This, too, is justified for indium and thallium, since both elements donate their outer $p$ electron to graphene and form nonmagnetic configurations. In a perturbative tight-binding picture, electrons hopping onto the adatom from graphene therefore lead to a singly occupied $p$ orbital, for which Coulomb repulsion is expected to be unimportant. Finally, we note that $H_{a}$ is even under $R_{z}$ symmetry even though we did not enforce this. In other words, the fact that the adatom is displaced away from the graphene sheet leads to no additional terms in the Hamiltonian $H_{a}$ compared to the (fictitious) case for an H-position adatom residing directly in the $(x, y)$ plane. This fact is crucial for the irrelevance of induced Rashba terms for graphene, as we discuss further below.

For the graphene-only part of the Hamiltonian, we posit that the usual nearest-neighbor hopping model $H_{t}$ is modified primarily through a change in chemical potential for the six sites adjacent to the adatom:

$$
H_{g}=H_{t}-\delta \mu \sum_{j=1}^{6} c_{\mathbf{r}_{j}}^{\dagger} c_{\mathbf{r}_{j}} .
$$

Clearly $H_{g}$ is invariant under not only $R_{\pi / 3}, R_{x}$, and $\mathcal{T}$, but also under $R_{z}$, despite this symmetry's being broken by the adatom. (Terms that violate $R_{z}$ necessarily involve spinorbit coupling, which we have justifiably neglected because it is exceedingly weak.)

Since the adatom generically modifies the bond lengths between carbon atoms in its vicinity, the hopping amplitudes will be modulated as well near the adatom. According to our DFT calculations, however, perturbations to graphene's lattice structure are quite small. (The carbon bond lengths in the immediate vicinity of either indium or thallium change by less than $1 \%$.) We thus expect this effect to be minor and have neglected it for simplicity. We have also neglected in $H_{g}$ any spin-orbit terms that directly couple the carbon atoms, which are orders of magnitude weaker than the spin-orbit interaction indirectly mediated by the adatom.

In contrast, the induced chemical potential $\delta \mu$ is not a weak effect-we find that our tight-binding simulations best reproduce the DFT band structure when $\delta \mu$ is on the order of $1 \mathrm{eV}$. Physically, $\delta \mu$ appears because the indium or thallium adatoms donate their outer $p$-orbital electron to the graphene sheet, leaving behind a net positive charge. Electrons in the graphene sheet thus prefer to conglomerate in its vicinity in order to screen the positively charged adatom. We note that, of course, there is no fundamental reason why the induced chemical potential should be confined only to the six sites nearest to the adatom. Our DFT simulations demonstrate, however, that the induced charge modulation occurs very locally around the adatom, so that this is expected to be a good approximation. As an illustration, consider the geometry with one thallium adatom in a $4 \times 4$ supercell. Figure 5 displays the induced charge density $\quad \Delta \rho \equiv \rho$ (graphene+thallium $)-\rho$ (graphene $)-$ $\rho$ (thallium) relative to the charge densities obtained when graphene and thallium decouple completely. Here, the yellow and blue regions correspond to isosurfaces with $\Delta \rho= \pm 0.01 \mathrm{eV} / \AA^{3}$. Clearly, the charge modulation occurs predominantly within the first two "rings" of carbon sites surrounding the adatom.

Finally, let us discuss the Hamiltonian $H_{c}$ that allows electrons to tunnel between the adatom and the graphene sheet. For simplicity, we allow only for tunneling events

(a)

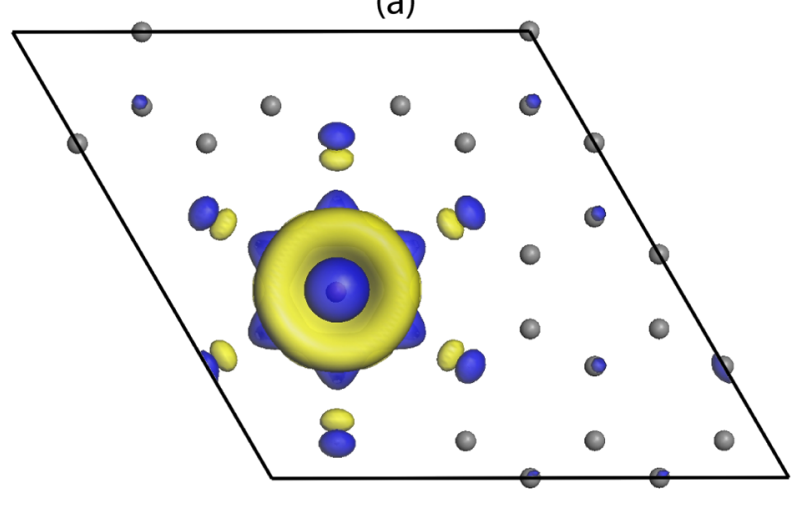

(b)

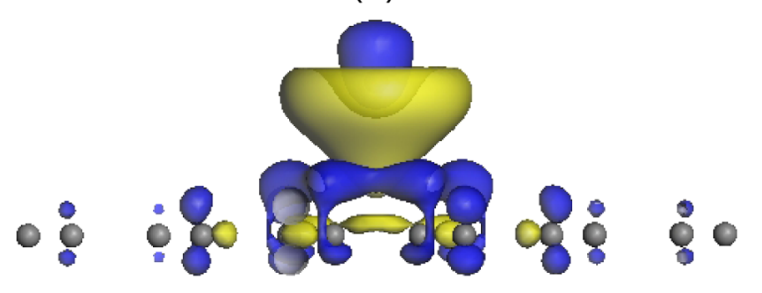

FIG. 5. Adatom-induced charge modulation. (a) Top and (b) side views of the charge density induced by a thallium adatom in a $4 \times 4$ supercell. Yellow (blue) surfaces correspond to a positive (negative) induced charge density (relative to the case where graphene and thallium decouple completely). The charge modulations relax rather rapidly away from the adatom and occur mostly within the two innermost "rings" of carbon sites near the adatom. 
that couple the adatom to its six nearest carbon atoms, and we assume (because of carbon's weak intrinsic spin-orbit coupling) that such processes are spin-independent. As in Eq. (3), it is useful to introduce linear combinations

$$
C_{m \alpha}=\frac{1}{\sqrt{6}} \sum_{j=1}^{6} e^{-i(\pi / 3) m(j-1)} c_{\mathbf{r}_{j} \alpha}
$$

that, like $d_{m \alpha}$, carry angular-momentum quantum number $m$ under $R_{\pi / 3}$. It follows from the symmetrytransformation rules for $c_{\mathbf{r}}$ obtained above that $C_{m \alpha}$ transforms as follows:

$$
\begin{gathered}
R_{\pi / 3}: C_{m} \rightarrow e^{i(\pi / 6) s^{z}} e^{i(\pi / 3) m} C_{m}, \\
R_{x}: C_{m} \rightarrow e^{i(\pi / 2) s^{x}} C_{-m}, \\
\mathcal{T}: C_{m} \rightarrow e^{i(\pi / 2) s^{y}} C_{-m}, \\
R_{z}: C_{m} \rightarrow-e^{i(\pi / 2) s^{z}} C_{m} .
\end{gathered}
$$

Using the first three symmetries along with the corresponding transformation rules for $d_{m}$, it is straightforward to show that the spin-independent hoppings of interest must take the form

$$
H_{c}=-\sum_{m=0, \pm 1}\left(t_{|m|} C_{m}^{\dagger} d_{m}+\text { H.c. }\right),
$$

with $t_{0}$ real and $t_{1}$ pure imaginary. Under $R_{z}$ symmetry, the $t_{0}$ hopping is even, while the $t_{1}$ hopping terms are odd. This is easy to understand physically by considering the fictitious case where the adatom resides exactly in the graphene plane so that $R_{z}$ symmetry is present. In this case, the $t_{1}$ terms reflect hopping between the adatom's $p_{x, y}$ orbitals and the carbon $p_{z}$ orbitals, which are orthogonal in this pathological limit. That is, tunneling between graphene's $p_{z}$ orbitals and the adatom's $m= \pm 1$ orbitals arises only when $R_{z}$ is lifted. In contrast, the adatom's $p_{z}$ orbitals overlap nontrivially with carbon's, even when $R_{z}$ is preserved.

Remarkably, the only terms in $H$ that do not respect $R_{z}$ are precisely these $t_{1}$ couplings. To see what kind of spinorbit processes the adatom can mediate, let us now consider the coupling between graphene and the adatom from a perturbative perspective. An electron from the graphene sheet can tunnel onto the adatom via either $t_{0}$ or $t_{1}$. Because of the adatom's strong spin-orbit coupling, the electron need not return to the graphene sheet via the same tunneling process by which it arrived. For example, an electron can tunnel via the $t_{0}$ hopping, flip its spin, and then return to the graphene sheet via $t_{1}$. Consequently, at second order in perturbation theory, the adatom will mediate effective couplings between the carbon atoms that are proportional to $t_{0}^{2}, t_{1}^{2}$, and $t_{0} t_{1}$. The electron's $S^{z}$ spin component is conserved in the former two cases, but not in the third. Furthermore, since the $t_{0}$ coupling in $H_{c}$ is even under $R_{z}$ while the $t_{1}$ coupling is odd, it is only the induced terms for graphene proportional to $t_{0} t_{1}$ that violate $R_{z}$. It follows that such spin-flip processes mediate Rashba spin-orbit coupling, while the $t_{1}^{2}$ events mediate intrinsic spin-orbit interactions. (The $t_{0}^{2}$ processes do not generate spin-orbit coupling.) It is worth noting as an aside that our ability to classify such processes in this simple way relies on the fact that all terms in $H_{a}$ are even under $R_{z}$. If $H_{a}$ also involved terms that violated $R_{z}$, then any second-order tunneling event would generically be capable of mediating Rashba spin-orbit coupling.

As discussed in the main text, to generate a QSH state in graphene, the physics near the Dirac points must be dominated by intrinsic rather than Rashba spin-orbit interactions. An important feature of our proposal is that Rashba coupling mediated by an adatom with a partially filled $p$ shell is always subdominant to the induced intrinsic spinorbit terms. This follows because electrons with momentum near the Dirac points hop very ineffectively onto the adatom's $m=0$ orbital. Previously we discussed how this effect arises because electrons destructively interfere when tunneling onto this orbital. Here we provide a complementary perspective, using the language of low-energy continuum Dirac fields. Focusing on physics near the Dirac points, one can express the lattice fermion operators for the $\mathrm{A}$ and $\mathrm{B}$ sublattices of graphene as follows:

$$
\begin{aligned}
& c_{\mathbf{r} \in A, \alpha} \sim e^{i \mathbf{Q} \cdot \mathbf{r}} \psi_{R, A \alpha}+e^{-i \mathbf{Q} \cdot \mathbf{r}} \psi_{L, A \alpha}, \\
& c_{\mathbf{r} \in B, \alpha} \sim e^{i \mathbf{Q} \cdot \mathbf{r}} \psi_{R, B \alpha}+e^{-i \mathbf{Q} \cdot \mathbf{r}} \psi_{L, B \alpha} .
\end{aligned}
$$

Here, $\psi_{R / L \alpha}$ are slowly varying, two-component fields that describe low-energy excitations in the vicinity of the Dirac points at momenta $\pm \mathbf{Q}= \pm \frac{4 \pi}{3 \sqrt{3} a} \hat{\mathbf{x}}$. ( $a$ is the separation between neighboring carbon atoms.) Let us use this decomposition to rewrite the operator $C_{m=0}$ that appears in the $t_{0}$ tunneling. Neglecting pieces involving derivatives of $\psi_{R / L \alpha}$, we obtain

$$
\begin{aligned}
C_{m=0} \sim & \frac{1}{\sqrt{6}}\left[\left(e^{i \mathbf{Q} \cdot \mathbf{r}_{2}}+e^{i \mathbf{Q} \cdot \mathbf{r}_{4}}+e^{i \mathbf{Q} \cdot \mathbf{r}_{6}}\right) \psi_{R, A}+\left(e^{i \mathbf{Q} \cdot \mathbf{r}_{1}}+e^{i \mathbf{Q} \cdot \mathbf{r}_{3}}\right.\right. \\
& \left.+e^{i \mathbf{Q} \cdot \mathbf{r}_{5}}\right) \psi_{R, B}+\left(e^{-i \mathbf{Q} \cdot \mathbf{r}_{2}}+e^{-i \mathbf{Q} \cdot \mathbf{r}_{4}}+e^{-i \mathbf{Q} \cdot \mathbf{r}_{6}}\right) \psi_{L, A} \\
& \left.+\left(e^{-i \mathbf{Q} \cdot \mathbf{r}_{1}}+e^{-i \mathbf{Q} \cdot \mathbf{r}_{3}}+e^{-i \mathbf{Q} \cdot \mathbf{r}_{5}}\right) \psi_{L, B}\right] .
\end{aligned}
$$

Remarkably, all terms in parentheses above vanish identically. The leading terms in the low-energy expansion of $C_{m=0}$ thus involve derivatives and become, consequently, increasingly unimportant as one approaches the Dirac points.

This implies that the Rashba coupling mediated by the adatom-which necessarily involves tunneling via $t_{0}$-is highly ineffective at influencing the physics at the Dirac points. In contrast, $C_{m= \pm 1}$ admits a nontrivial expansion in terms of $\psi_{R / L \alpha}$ even when terms involving derivatives are dropped. It follows that electrons with momenta near the Dirac points can effectively tunnel onto and off the adatom 
via $t_{1}$, and the intrinsic spin-orbit coupling mediated by these hoppings thus always dominates over the induced Rashba terms. As noted in the main text, this is not necessarily the case for adatoms with partially filled $d$ or $f$ shells, because there are then additional orbitals onto which electrons from graphene can tunnel. For instance, here, electrons can tunnel onto the adatom's $m= \pm 2$ orbitals via a tunneling $t_{2}$; such processes, unlike $t_{0}$ tunneling, are not suppressed at the Dirac points. Effective couplings between carbon atoms proportional to $t_{1} t_{2}$ are odd under $R_{z}$, involve spin flips, and correspond to Rashba spin-orbit interactions that may significantly affect the low-energy physics at the Dirac points. The importance of such terms compared to the induced intrinsic spin-orbit interactions then depends on details of the Hamiltonian. If, say, the additional orbitals hybridize very weakly with graphene or reside far in energy from the Dirac points due to crystal-field effects, then $d$ - or $f$-shell adatoms may still be suitable for stabilizing a QSH state in graphene.

\section{APPENDIX B: DENSITY FUNCTIONAL THEORY}

All DFT calculations were carried out with the Vienna ab-initio simulation package (VASP) [45,46], at the level of the generalized-gradient approximation (GGA) [47]. We used the projector augmented wave method for the description of the core-valence interaction [48,49]. A vacuum space of $15 \AA$ was employed in all calculations, along with a $15 \times 15 k$-grid mesh [50] for integrals in the twodimensional Brillouin zone. The energy cutoff of the plane wave expansion was set to $500 \mathrm{eV}$.

For pure graphene, the optimized lattice constant is $2.468 \AA$, consistent with the experimental value of $2.46 \AA$. When addressing the preferred location $(\mathrm{H}, \mathrm{B}$, or $\mathrm{T}$ ) of different elements on graphene, a $4 \times 4$ supercell was employed with one adatom per cell. Recall that we have two minimal criteria for stabilizing a QSH state in graphene: the adatoms should (i) prefer to occupy the $\mathrm{H}$ position and (ii) not form a net spin moment. Among the elements we searched in this work, In, I, La, Hf, Au, Hg and $\mathrm{Tl}$ satisfy the first criterion, while the remaining elements prefer either the T or B site. However, I, La, Hf and $\mathrm{Au}$ form magnetic moments and thus fail the second criterion, while the $s$ and $d$ bands of $\mathrm{Hg}$ reside far below graphene's Fermi level, implying a negligible interaction between the two. The only two remaining elementsindium and thallium-satisfy both criteria and enter the $\mathrm{H}$ site nonmagnetically.

Including spin-orbit coupling, the binding energies $\left[E_{b}=E(\right.$ adatom+graphene $)-E($ graphene $)-E($ atom $\left.)\right]$ of indium and thallium on graphene at the $\mathrm{H}$ site are -0.525 and $-0.133 \mathrm{eV}$, respectively. The total energy per site when indium resides at the $\mathrm{T}$ and $\mathrm{B}$ sites is higher by 87 and $80 \mathrm{meV}$, respectively, than when indium resides at the $\mathrm{H}$ site. This is qualitatively consistent with the results of Ref. [20], where spin-orbit coupling was neglected. The conclusion is the same for thallium, although the preference for the $\mathrm{H}$ position is weaker; here, the $\mathrm{T}$ and $\mathrm{B}$ sites are higher in energy by 32 and $22 \mathrm{meV}$. At low temperatures compared to these energy differences, indium and thallium are expected to occupy only the $\mathrm{H}$ position, with indiumcarbon and thallium-carbon bond lengths of 2.83 and $2.90 \AA$, respectively, as determined by GGA calculations. We also calculated the atomic structures of indium and thallium on graphene within the local density approximation. The indium-carbon and thallium-carbon bonds are shortened by only 0.09 and $0.06 \AA$, respectively, compared with the GGA results. It is thus reasonable to conclude that both GGA and the local density approximation can describe the structural and electronic properties well.

\section{APPENDIX C: ADIABATIC CONTINUITY OF THE ADATOM AND KANE-MELE MODELS}

We now turn to a periodic system with one indium or thallium adatom in a $4 \times 4$ supercell. This problem was explored extensively in the main text using both DFT and tight-binding simulations. Our first-principles calculations demonstrated that, with either element, the Dirac points remain massless in the nonrelativistic limit but acquire a substantial gap upon including spin-orbit coupling. We further showed that the band structure and local density of states at the adatom site could be well-accounted for by an effective tight-binding model $H_{4 \times 4}$. This model included first-, second-, and third-neighbor hopping amongst carbon atoms in the graphene sheet, as well as the additional couplings discussed in Sec. II around each adatom. Note that inclusion of these further-neighbor tunnelings is necessary for obtaining reasonable quantitative agreement with first-principles band structure at energies away from the Dirac points, even for pure graphene [51]. Since the adatoms' local density of states is concentrated at energies greater than $1 \mathrm{eV}$ above the Dirac points (see Fig. 2), one needs these additional hoppings to accurately model their hybridization with the graphene bands. (We stress that this is important for a quantitative description only. None of the qualitative features discussed in this paper depends on these fine details. For instance, with only nearest-neighbor hopping, a gap still opens at the Dirac points, although the local density of states for the adatoms' $p_{z}$ states is difficult to accurately fit in this case.)

One advantage of this effective tight-binding model is that it enables one to readily verify that the insulating regime generated by the spin-orbit-induced gap indeed corresponds to a QSH state. This can perhaps be most easily proven by demonstrating that $H_{4 \times 4}$ and the Kane-Mele model $H_{\mathrm{KM}}$ [see Eqs. (1) and (2)] can be smoothly deformed into one another without closing the Dirac-point gap. To this end, consider the Hamiltonian $H(\lambda)=$ $(1-\lambda) H_{4 \times 4}+\lambda H_{\mathrm{KM}}$, introduced in Sec. III, that interpolates between these models as $\lambda$ varies from 0 to 1 . For $H_{\mathrm{KM}}$ 


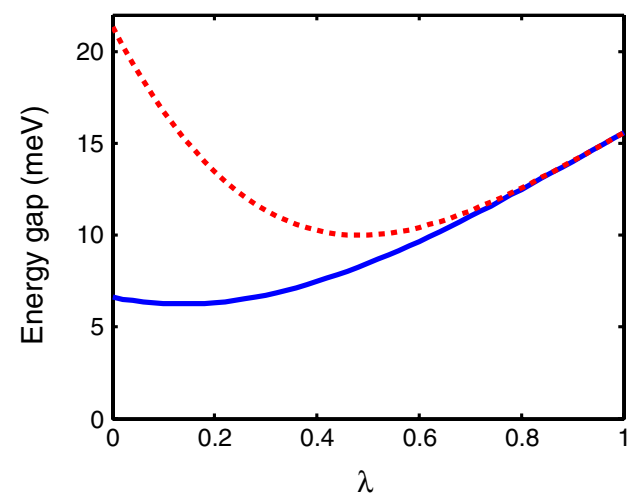

FIG. 6. Adiabatic continuity. Energy gap at the Dirac point as the Hamiltonian adiabatically deforms from the periodic adatom Hamiltonian $H_{4 \times 4}$ at $\lambda=0$ to the Kane-Mele model at $\lambda=1$. The solid blue and dashed red lines correspond to $H_{4 \times 4}$ evaluated with parameters appropriate for indium and thallium, respectively. In both cases, the gap remains finite as $\lambda$ varies from 0 to 1 , indicating that the Kane-Mele model and adatom Hamiltonian are adiabatically connected and thus support the same quantum spin-Hall phase.

(which includes only nearest-neighbor hopping and uniform intrinsic spin-orbit coupling for graphene), we choose $t=2.7 \mathrm{eV}$ and $\lambda_{\mathrm{so}}=-0.0015 \mathrm{eV}$. As Fig. 6 illustrates, the Dirac-point gap computed for $H(\lambda)$ indeed remains finite for all $\lambda$ between 0 to 1 with either indium (solid blue line) or thallium (dashed red line) parameters input into $H_{4 \times 4}$. The QSH state known to be supported by $H_{\mathrm{KM}}$ and the insulating state stabilized by either type of adatom are, consequently, the same topological phase of matter.

\section{APPENDIX D: GRAPHENE-ONLY EFFECTIVE HAMILTONIAN}

In this section, we outline the derivation of the grapheneonly Hamiltonian, Eqs. (8) and (9), from the tight-binding model in Eqs. (4)-(7) describing the graphene sheet with H-position adatoms. We assume, for the purposes of this section only, that one adatom resides at each hexagonal plaquette but disallow any direct hopping of electrons between adatom $p$ orbitals. Under these assumptions we may exploit the translation symmetry of the problem and write the Hamiltonian (4) in momentum space as

$$
H=\sum_{\mathbf{k}} \psi_{\mathbf{k}}^{\dagger} \mathcal{H}_{\mathbf{k}} \psi_{\mathbf{k}}
$$

with $\psi_{\mathbf{k}}=\left(c_{\mathbf{k}}^{A}, c_{\mathbf{k}}^{B} ; d_{1 \mathbf{k}}, d_{0 \mathbf{k}}, d_{-1 \mathbf{k}}\right)^{T}$, where $c_{\mathbf{k}}^{A / B}$ remove electrons from the A/B graphene sublattices while $d_{m \mathbf{k}}$ remove electrons from the adatom orbitals. As before, spin is treated implicitly. The Hamiltonian matrix in this basis reads

$$
\mathcal{H}_{\mathbf{k}}=\left(\begin{array}{cc}
h_{g} & T \\
T^{\dagger} & h_{a}
\end{array}\right)
$$

where

$$
h_{g}=\left(\begin{array}{cc}
0 & \tau_{\mathbf{k}} \\
\tau_{\mathbf{k}}^{*} & 0
\end{array}\right), \quad \tau_{\mathbf{k}}=-t \sum_{j=0}^{2} e^{i \mathbf{k} \cdot \boldsymbol{\delta}_{j}}
$$

is the standard nearest-neighbor tight-binding Hamiltonian for graphene with $\boldsymbol{\delta}_{j}=\left(\sin \frac{2 \pi j}{3},-\cos \frac{2 \pi j}{3}\right)$ unit vectors pointing from a site on sublattice $A$ to its three neighbors on the B sublattice. Also,

$$
h_{a}=\left(\begin{array}{ccc}
\epsilon_{1}+\Lambda_{\mathrm{so}} s^{z} & \sqrt{2} \Lambda_{\mathrm{so}}^{\prime} s^{-} & 0 \\
\sqrt{2} \Lambda_{\mathrm{so}}^{\prime} s^{+} & \epsilon_{0} & \sqrt{2} \Lambda_{\mathrm{so}}^{\prime} s^{-} \\
0 & \sqrt{2} \Lambda_{\mathrm{so}}^{\prime} s^{+} & \epsilon_{1}-\Lambda_{\mathrm{so}} s^{z}
\end{array}\right)
$$

describes the adatom $p$ orbitals. Finally,

$T=\left(\begin{array}{ccc}V_{1} & V_{0} & V_{-1} \\ V_{-1}^{*} & V_{0}^{*} & V_{1}^{*}\end{array}\right), \quad V_{m}=t_{|m|} \sum_{j=0}^{2} e^{i(2 \pi j / 3) m} e^{-i \mathbf{k} \cdot \boldsymbol{\delta}_{j}}$

represent transitions between graphene and the adatom orbitals as described by $H_{c}$ in Eq. (7).

We now wish to "integrate out" the adatom degrees of freedom and thus determine their effect on the electrons in graphene. The simplest way to accomplish this goal is to first perform a unitary transformation $\mathcal{H} \rightarrow \tilde{\mathcal{H}}=$ $e^{-S} \mathcal{H} e^{S}$, with $S$ chosen such that $\tilde{h}_{g}$ and $\tilde{h}_{a}$ in the transformed Hamiltonian are decoupled, i.e., $\tilde{T}=0$. In this basis, the adatom degrees of freedom can be integrated out trivially. Following the steps outlined in Ref. [9], we find, to second order in $T$,

$\tilde{h}_{g}=h_{g}-\frac{1}{2}\left[T h_{a}^{-1} T^{\dagger}+h_{g} T h_{a}^{-2} T^{\dagger}+\right.$ H.c. $]+\mathcal{O}\left(T^{4}\right)$.

This result is equivalent to treating Hamiltonian (D1) perturbatively to second order in $T$.

We are interested in the effect of adatoms on the lowenergy fermonic modes in graphene occurring in the vicinity of the Dirac points $\pm \mathbf{Q}$. In this limit, clearly, the second term in the brackets becomes negligible (since $h_{g}$ vanishes as $\mathbf{k} \rightarrow \pm \mathbf{Q}$ ), so we therefore focus on the first term. Although it is possible to find an exact inverse of $h_{a}$, the result is cumbersome and tends to obscure the simple physics underlying the formation of the spin-orbit-induced gap in the system. To avoid this complication, we make an additional assumption that spin-orbit splitting of the adatom orbitals is small compared to the crystal-field effects, $\left|\Lambda_{\mathrm{so}}\right|,\left|\Lambda_{\mathrm{so}}^{\prime}\right| \ll\left|\epsilon_{m}\right|$, and write $h_{a}=h_{0}+h_{1}$ with $h_{0}=$ $\operatorname{diag}\left(\epsilon_{1}, \epsilon_{0}, \epsilon_{1}\right)$. To first order in $\Lambda_{\text {so }}$ and $\Lambda_{\text {so }}^{\prime}$ we then have $h_{a}^{-1} \simeq h_{0}^{-1}-h_{0}^{-1} h_{1} h_{0}^{-1}$ and

$$
\tilde{h}_{g} \simeq h_{g}-T h_{0}^{-1} T^{\dagger}+T h_{0}^{-1} h_{1} h_{0}^{-1} T^{\dagger} .
$$

The adatom-induced correction to $h_{g}$ is seen to have a simple structure. The first term is spin-independent and mediates on-site potential terms and hoppings between sites in the hexagon surrounding an adatom. The second, spin-dependent term in turn produces the intrinsic and Rashba spin-orbit couplings. 
Various terms resulting from Eq. (D6) can be explicitly worked out in a somewhat tedious but straightforward calculation. The spin-orbit terms take the form

$$
\delta h_{g}^{\mathrm{SOC}}=\left(\begin{array}{cc}
R_{1}+D & R_{2} \\
R_{2}^{\dagger} & R_{1}-D
\end{array}\right)
$$

with

$$
D=\Lambda_{\mathrm{so}} \frac{\left|t_{1}\right|^{2}}{\sqrt{3} \epsilon_{1}^{2}} s^{z} \sum_{j=0}^{2} \sin \mathbf{k} \cdot\left(\boldsymbol{\delta}_{j}-\boldsymbol{\delta}_{j+1}\right)
$$

and

$$
\begin{aligned}
& R_{1}=\Lambda_{\mathrm{so}}^{\prime} \frac{\sqrt{2} t_{0}\left|t_{1}\right|}{3 \epsilon_{0} \epsilon_{1}} \sum_{j, l}\left[\mathbf{s} \times\left(\boldsymbol{\delta}_{l}-\boldsymbol{\delta}_{j}\right)\right]_{z} \sin \mathbf{k} \cdot\left(\boldsymbol{\delta}_{j}-\boldsymbol{\delta}_{l}\right), \\
& R_{2}=-i \Lambda_{\mathrm{so}}^{\prime} \frac{\sqrt{2} t_{0}\left|t_{1}\right|}{3 \epsilon_{0} \epsilon_{1}} \sum_{j, l}\left[\mathbf{s} \times\left(\boldsymbol{\delta}_{l}+\boldsymbol{\delta}_{j}\right)\right]_{z} e^{-i \mathbf{k} \cdot\left(\boldsymbol{\delta}_{j}+\boldsymbol{\delta}_{l}\right)} .
\end{aligned}
$$

If we now recall that $\boldsymbol{\delta}_{0}+\boldsymbol{\delta}_{1}+\boldsymbol{\delta}_{2}=0$ and identify

$$
\lambda_{\mathrm{so}}=\Lambda_{\mathrm{so}} \frac{\left|t_{1}\right|^{2}}{2 \sqrt{3} \epsilon_{1}^{2}}, \quad \lambda_{R}=\Lambda_{\mathrm{so}}^{\prime} \frac{\sqrt{2} t_{0}\left|t_{1}\right|}{3 \epsilon_{0} \epsilon_{1}},
$$

we see that $D$ and $R_{1,2}$ correspond, respectively, to the intrinsic and hexagon Rashba spin-orbit couplings in Eq. (9). One can readily verify that $R_{1,2}$ vanish at $\mathbf{k}=$ $\pm \mathbf{Q}$ while $D$ approaches a nonzero value of $3 \sqrt{3} \lambda_{\text {so }}$, as expected on the basis of the arguments presented above.

Using the tight-binding parameters obtained in Sec. III, we may estimate $\lambda_{\mathrm{so}} \simeq 23 \mathrm{meV}$ and $\lambda_{R}=58 \mathrm{meV}$ for thallium. (For indium, both are about a factor of 3 smaller.) At $6 \%$ coverage, assuming uniform averaging of $\lambda_{\text {so }}$ over all plaquettes, this would imply a spin-orbit-induced gap of $0.06 \times 6 \sqrt{3} \lambda_{\mathrm{so}} \simeq 14 \mathrm{meV}$, somewhat smaller that the $21 \mathrm{meV}$ gap predicted by DFT at the same coverage. We attribute this discrepancy to the neglect of higher-order terms in Eq. (D6); since $t_{0,1}$ are of similar magnitude as $\epsilon_{0,1}$, this expansion cannot be expected to yield quantitatively accurate values of the coupling constants.
Nevertheless, the procedure outlined above is useful in that it illustrates how various symmetry-allowed spin-orbit terms emerge in the effective graphene-only model. It also confirms that, although the Rashba coupling exceeds the intrinsic coupling by more than a factor of 2 , its special form, dictated by symmetry, forces it to vanish at the Dirac point and renders $\lambda_{R}$ irrelevant for the low-energy physics.

Using the same methods, one can also estimate various spin-independent terms induced by the adatoms. These include the on-site potential and additional hopping terms mentioned above. Since the latter are already present in pristine graphene and since adatom corrections are generally small compared to these bare values, we expect their effect on the low-energy physics to be minimal and do not discuss them further.

\section{APPENDIX E: HEXAGONAL RASHBA AND DISORDER EFFECTS}

As mentioned in Sec. VI, the stability of the QSH phase was also tested against the addition of hexagonal Rashba coupling and residual disorder in the graphene sheet. We now discuss these results in greater detail. Throughout this appendix, we will consider random-adatom arrangements at coverage $n_{i}=0.2$, modeled using the graphene-only Hamiltonian from Eq. (8) with $\delta \mu=0$ for simplicity. We first consider the influence of the hexagon Rashba term defined in Eq. (9). While it is true that its effects identically vanish at the Dirac points, it nevertheless causes some splitting of the bands in their vicinity. As a result, these Rashba terms can reduce somewhat the size of the mobility gap observed with either periodic or random adatoms, although to a far lesser extent than the traditional Rashba coupling. The splitting will depend on the ratio of $\lambda_{\text {so }}$ to $\lambda_{R}$, and, more importantly, the absolute strength of $\lambda_{R}$ compared to $t$.

In Fig. 7(a), we show the result for a set of parameters chosen to illustrate this effect while avoiding the compli-
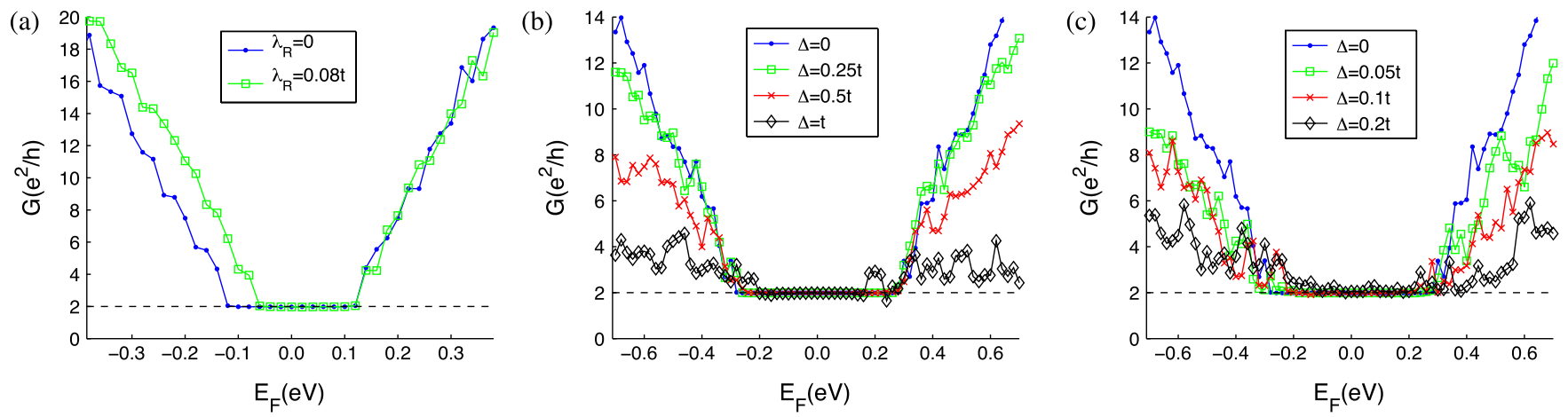

FIG. 7. Hexagonal Rashba effect and substrate-induced disorder. Conductance $G$ as a function of the Fermi energy $E_{\mathrm{F}}$ for a system including (a) hexagonal Rashba coupling, (b) uncorrelated disorder randomly distributed over the range $[-\Delta, \Delta]$, and (c) correlated disorder as defined in Eq. (E1) with $\rho=0.07$ and $d=5$. In all cases, the system is of size $W=80$ and $L=40$ with adatom coverage $n_{i}=0.2$ and nearest-neighbor hopping strength $t=2.7 \mathrm{eV}$. For panel (a), the intrinsic spin-orbit strength is $\lambda_{\text {so }}=0.04 t$; for panels (b) and (c), $\lambda_{\text {so }}=0.1 t$. 
cations due to the finite size of the system. Here, $\lambda_{\text {so }}=$ $0.04 t$ and $\lambda_{R}=0.08 t$, their ratio chosen to be similar to that estimated in Eq. (D9). The mobility gap has clearly been somewhat reduced, but a robust plateau remains. We remark that the standard Rashba term (with only nearestneighbor coupling) at the same coupling strength would completely destroy the plateau. For smaller values of $\lambda_{R}$ and $\lambda_{\text {so }}$ (but keeping their ratio constant), the effect of hexagonal Rashba coupling becomes even less pronounced, although the finite-size effects prevent us from obtaining reliable results at low-adatom coverage in this regime. It is also worth emphasizing that one should not view $\lambda_{R}$ as reducing the gap compared to those found in our DFT and tight-binding results displayed in Fig. 2. Those simulations incorporated the intrinsic and the hexagon Rashba spin-orbit couplings (among other couplings neglected for simplicity here), so the results already reflect the influence of both types of spin-orbit interactions.

To study residual disorder unrelated to the adatoms (e.g., arising from impurities in the substrate), two models were used, both of which can be captured by an on-site potential term of the form specified in Eq. (8). In the first model, we incorporate an uncorrelated random chemical potential in the range $[-\Delta, \Delta]$ on every lattice site. In the second, we employ a longer-range, correlated disorder potential of the form [52]

$$
\delta \mu_{\mathbf{r}_{i}}=\sum_{j=1}^{N_{\mathrm{imp}}} V_{j} \exp \left(-\frac{\left|\mathbf{r}_{i}-\mathbf{r}_{j}\right|^{2}}{2 d^{2}}\right),
$$

where $N_{\text {imp }}$ is the number of impurity sites, $V_{j}$ is also uniformly random over the range $[-\Delta, \Delta]$, and $d$ is the radius of the impurity potential. As in Ref. [52], we characterize $N_{\text {imp }}$ by the ratio $\rho=N_{\text {imp }} / N$, with $N$ being the total number of carbon atoms in the sample.

While we have not averaged over multiple disorder realizations as in Fig. 4(b), with only a single disorder realization, one can still see the general trend toward instability of the QSH phase as $\Delta$ increases. Our simulations show that this state, despite already being stabilized by a disordered arrangement of adatoms, in fact exhibits remarkable resilience against additional uncorrelated substrate-induced disorder in the graphene sheet. As Fig. 7(b) illustrates, in this case, a very high value of $\Delta \approx t$ can be applied before the first signs of disorder affecting the topological phase appear, and even here a considerable conductance plateau survives. The topological phase is, however, more sensitive to long-range correlated disorder, which is likely more relevant for experiment when charged impurities from the substrate present the main disordering mechanism. Figure 7(c) illustrates that, here, the deterioration of the plateau is more rapid. At $\Delta=$ $0.2 t \approx 0.54 \mathrm{eV}$, with $\rho=0.07$ and $d=5$, there is still a remnant of the gapless edge states, but, for stronger disorder, the topological phase is destroyed. Notice that this value of $\Delta$ is comparable to the width of the plateau in the "clean" case, where the only disorder source is the randomly distributed adatoms.

[1] Joel E. Moore, The Birth of Topological Insulators, Nature (London) 464, 194 (2010).

[2] M.Z. Hasan and C.L. Kane, Colloquium: Topological Insulators, Rev. Mod. Phys. 82, 3045 (2010).

[3] Xiao-Liang Qi and Shou-Cheng Zhang, Topological Insulators and Superconductors, arXiv:1008.2026 [Rev. Mod. Phys. (to be published)].

[4] Liang $\mathrm{Fu}$ and C.L. Kane, Josephson Current and Noise at a Superconductor/Quantum-Spin-Hall-Insulator/ Superconductor Junction, Phys. Rev. B 79, 161408 (2009).

[5] Chetan Nayak, Steven H. Simon, Ady Stern, Michael Freedman, and Sankar Das Sarma, Non-Abelian Anyons and Topological Quantum Computation, Rev. Mod. Phys. 80, 1083 (2008).

[6] C. L. Kane and E. J. Mele, Quantum Spin Hall Effect in Graphene, Phys. Rev. Lett. 95, 226801 (2005).

[7] Daniel Huertas-Hernando, F. Guinea, and Arne Brataas, Spin-Orbit Coupling in Curved Graphene, Fullerenes, Nanotubes, and Nanotube Caps, Phys. Rev. B 74, 155426 (2006).

[8] Hongki Min, J. E. Hill, N. A. Sinitsyn, B. R. Sahu, Leonard Kleinman, and A.H. MacDonald, Intrinsic and Rashba Spin-Orbit Interactions in Graphene Sheets, Phys. Rev. B 74, 165310 (2006).

[9] Yugui Yao, Fei Ye, Xiao-Liang Qi, Shou-Cheng Zhang, and Zhong Fang, Spin-Orbit Gap of Graphene: FirstPrinciples Calculations, Phys. Rev. B 75, 041401 (2007).

[10] J.C. Boettger and S.B. Trickey, First-Principles Calculation of the Spin-Orbit Splitting in Graphene, Phys. Rev. B 75, 121402 (2007).

[11] M. Gmitra, S. Konschuh, C. Ertler, C. Ambrosch-Draxl, and J. Fabian, Band-Structure Topologies of Graphene: Spin-Orbit Coupling Effects from First Principles, Phys. Rev. B 80, 235431 (2009).

[12] B. Andrei Bernevig, Taylor L. Hughes, and Shou-Cheng Zhang, Quantum Spin Hall Effect and Topological Phase Transition in HgTe Quantum Wells, Science 314, 1757 (2006).

[13] Chaoxing Liu, Taylor L. Hughes, Xiao-Liang Qi, Kang Wang, and Shou-Cheng Zhang, Quantum Spin Hall Effect in Inverted Type-II Semiconductors, Phys. Rev. Lett. 100, 236601 (2008).

[14] Shuichi Murakami, Quantum Spin Hall Effect and Enhanced Magnetic Response by Spin-Orbit Coupling, Phys. Rev. Lett. 97, 236805 (2006).

[15] Markus Konig, Steffen Wiedmann, Christoph Brune, Andreas Roth, Hartmut Buhmann, Laurens W. Molenkamp, Xiao-Liang Qi, and Shou-Cheng Zhang, Quantum Spin Hall Insulator State in HgTe Quantum Wells, Science 318, 766 (2007).

[16] S. Raghu, Xiao-Liang Qi, C. Honerkamp, and ShouCheng Zhang, Topological Mott Insulators, Phys. Rev. Lett. 100, 156401 (2008). 
[17] C. Weeks and M. Franz, Interaction-Driven Instabilities of a Dirac Semimetal, Phys. Rev. B 81, 085105 (2010).

[18] T. Pereg-Barnea and G. Refael, Inducing Topological Order in a Honeycomb Lattice, arXiv:1011.5243.

[19] Jun-Ichi Inoue and Akihiro Tanaka, Photoinduced Transition between Conventional and Topological Insulators in Two-Dimensional Electronic Systems, Phys. Rev. Lett. 105, 017401 (2010).

[20] Kevin T. Chan, J. B. Neaton, and Marvin L. Cohen, FirstPrinciples Study of Metal Adatom Adsorption on Graphene, Phys. Rev. B 77, 235430 (2008).

[21] A. H. Castro Neto and F. Guinea, Impurity-Induced SpinOrbit Coupling in Graphene, Phys. Rev. Lett. 103, 026804 (2009).

[22] Samir Abdelouahed, A. Ernst, J. Henk, I. V. Maznichenko, and I. Mertig, Spin-Split Electronic States in Graphene: Effects Due to Lattice Deformation, Rashba Effect, and Adatoms by First Principles, Phys. Rev. B 82, 125424 (2010).

[23] Zhenhua Qiao, Shengyuan A. Yang, Wanxiang Feng, Wang-Kong Tse, Jun Ding, Yugui Yao, Jian Wang, and Qian Niu, Quantum Anomalous Hall Effect in Graphene from Rashba and Exchange Effects, Phys. Rev. B 82, 161414 (2010).

[24] R. Landauer, Electrical Resistance of Disordered OneDimensional Lattices, Philos. Mag. 21, 863 (1970).

[25] M. Büttiker, Absence of Backscattering in the Quantum Hall Effect in Multiprobe Conductors, Phys. Rev. B 38, 9375 (1988).

[26] G. Metalidis and P. Bruno, Green's Function Technique for Studying Electron Flow in Two-Dimensional Mesoscopic Samples, Phys. Rev. B 72, 235304 (2005).

[27] C. R. Dean, A. F. Young, I. Meric, C. Lee, L. Wang, S. Sorgenfrei, K. Watanabe, T. Taniguchi, P. Kim, K. L. Shepard, and J. Hone, Boron Nitride Substrates for High-Quality Graphene Electronics, Nature Nanotech. 5, 722 (2010).

[28] Jian Li, Rui-Lin Chu, J. K. Jain, and Shun-Qing Shen, Topological Anderson Insulator, Phys. Rev. Lett. 102, 136806 (2009).

[29] Hua Jiang, Lei Wang, Qing-feng Sun, and X.C. Xie, Numerical Study of the Topological Anderson Insulator in HgTe/CdTe Quantum Wells, Phys. Rev. B 80, 165316 (2009).

[30] C. W. Groth, M. Wimmer, A. R. Akhmerov, J. Tworzydło, and C.W.J. Beenakker, Theory of the Topological Anderson Insulator, Phys. Rev. Lett. 103, 196805 (2009).

[31] H.-M. Guo, G. Rosenberg, G. Refael, and M. Franz, Topological Anderson Insulator in Three Dimensions, Phys. Rev. Lett. 105, 216601 (2010).

[32] Emil Prodan, Three-Dimensional Phase Diagram of Disordered HgTe/CdTe Quantum Spin Hall Wells, Phys. Rev. B 83, 195119 (2011).

[33] A. Das, S. Pisana, B. Chakraborty, S. Piscanec, S. K. Saha, U. V. Waghmare, K.S. Novoselov, H. R. Krishnamurthy, A. K. Geim, A.C. Ferrari, and A. K. Sood, Monitoring Dopants by Raman Scattering in an Electrochemically TopGated Graphene Transistor, Nature Nanotech. 3, 210 (2008).

[34] Jun Yan, Theresa Villarson, Erik A. Henriksen, Philip Kim, and Aron Pinczuk, Optical Phonon Mixing in Bilayer Graphene with a Broken Inversion Symmetry, Phys. Rev. B 80, 241417 (2009).
[35] J. T. Ye, M.F. Craciun, M. Koshino, S. Russo, S. Inoue, H. T. Yuan, H. Shimotani, A. F. Morpurgo, and Y. Iwasa, Accessing the Transport Properties of Graphene and Its Multilayers at High Carrier Density, Proc. Natl. Acad. Sci. USA 108, 13002 (2011).

[36] Yi Zheng, Guang-Xin Ni, Sukang Bae, Chun-Xiao Cong, Orhan Kahya, Chee-Tat Toh, Hye Ri Kim, Danho Im, Ting $\mathrm{Yu}$, Jong Hyun Ahn, Byung Hee Hong, and Barbaros Özyilmaz, Wafer-Scale Graphene/Ferroelectric Hybrid Devices for Low-Voltage Electronics, Europhys. Lett. 93, 17002 (2011).

[37] Dmitri K. Efetov and Philip Kim, Controlling ElectronPhonon Interactions in Graphene at Ultrahigh Carrier Densities, Phys. Rev. Lett. 105, 256805 (2010).

[38] Andreas Roth, Christoph Brune, Hartmut Buhmann, Laurens W. Molenkamp, Joseph Maciejko, Xiao-Liang Qi, and Shou-Cheng Zhang, Nonlocal Transport in the Quantum Spin Hall State, Science 325, 294 (2009).

[39] G. Tkachov and E. M. Hankiewicz, Ballistic Quantum Spin Hall State and Enhanced Edge Backscattering in Strong Magnetic Fields, Phys. Rev. Lett. 104, 166803 (2010).

[40] Joseph Maciejko, Xiao-Liang Qi, and Shou-Cheng Zhang, Magnetoconductance of the Quantum Spin Hall State, Phys. Rev. B 82, 155310 (2010).

[41] Hubert B. Heersche, Pablo Jarillo-Herrero, Jeroen B. Oostinga, Lieven M. K. Vandersypen, and Alberto F. Morpurgo, Bipolar Supercurrent in Graphene, Nature (London) 446, 56 (2007).

[42] Xu Du, Ivan Skachko, and Eva Y. Andrei, Josephson Current and Multiple Andreev Reflections in Graphene SNS Junctions, Phys. Rev. B 77, 184507 (2008).

[43] C. Ojeda-Aristizabal, M. Ferrier, S. Guéron, and H. Bouchiat, Tuning the Proximity Effect in a Superconductor-Graphene-Superconductor Junction, Phys. Rev. B 79, 165436 (2009).

[44] Dongchan Jeong, Jae-Hyun Choi, Gil-Ho Lee, Sanghyun Jo, Yong-Joo Doh, and Hu-Jong Lee, Observation of Supercurrent in PbIn-Graphene-PbIn Josephson Junction, Phys. Rev. B 83, 094503 (2011).

[45] G. Kresse and J. Furthmuller, Efficiency of Ab-Initio Total Energy Calculations for Metals and Semiconductors Using a Plane-Wave Basis Set, Comput. Mater. Sci. 6, 15 (1996).

[46] G. Kresse and J. Furthmuller, Efficient Iterative Schemes for Ab Initio Total-Energy Calculations Using a PlaneWave Basis Set, Phys. Rev. B 54, 11169 (1996).

[47] J.P. Perdew, Kieron Burke, and Matthias Ernzerhof, Generalized Gradient Approximation Made Simple, Phys. Rev. Lett. 77, 3865 (1996).

[48] P.E. Blochl, Projector Augmented-Wave Method, Phys. Rev. B 50, 17953 (1994).

[49] G. Kresse and D. Joubert, From Ultrasoft Pseudopotentials to the Projector Augmented-Wave Method, Phys. Rev. B 59, 1758 (1999).

[50] H.J. Monkhorst and J.D. Pack, Special Points for Brillouin-Zone Integrations, Phys. Rev. B 13, 5188 (1976).

[51] S. Reich, J. Maultzsch, C. Thomsen, and P. Ordejón, TightBinding Description of Graphene, Phys. Rev. B 66, 035412 (2002).

[52] Shengjun Yuan, Hans De Raedt, and Mikhail I. Katsnelson, Electronic Transport in Disordered Bilayer and Trilayer Graphene, Phys. Rev. B 82, 235409 (2010). 